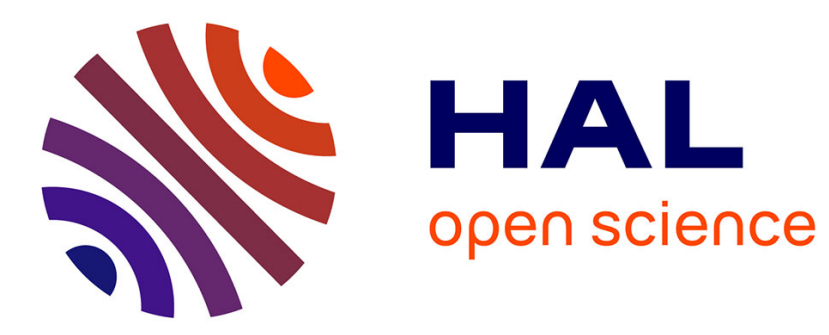

\title{
RANS modeling of Laminar Separation Bubbles around Airfoils at Low Reynolds conditions
}

\author{
Luis Bernardos, François Richez, Vincent Gleize
}

\section{To cite this version:}

Luis Bernardos, François Richez, Vincent Gleize. RANS modeling of Laminar Separation Bubbles around Airfoils at Low Reynolds conditions. AIAA AVIATION 2019, Jun 2019, DALLAS, United States. 10.2514/6.2019-2922 . hal-02333706

\section{HAL Id: hal-02333706 https://hal.science/hal-02333706}

Submitted on 25 Oct 2019

HAL is a multi-disciplinary open access archive for the deposit and dissemination of scientific research documents, whether they are published or not. The documents may come from teaching and research institutions in France or abroad, or from public or private research centers.
L'archive ouverte pluridisciplinaire HAL, est destinée au dépôt et à la diffusion de documents scientifiques de niveau recherche, publiés ou non, émanant des établissements d'enseignement et de recherche français ou étrangers, des laboratoires publics ou privés. 


\title{
RANS modeling of Laminar Separation Bubbles around Airfoils at Low Reynolds Number Conditions
}

\author{
L. Bernardos*, F. Richez ${ }^{\dagger}$ and V. Gleize \\ ONERA, 92190 Meudon, France
}

\begin{abstract}
In this paper we evaluate the performance of an algebraic non-local laminar separation transition triggering (LSTT) approach that controls the production of turbulence in the transitional region. The LSTT approach is coupled to the $k-\omega$ and Spalart-Allmaras turbulence models and the AHD-Gleyzes transition prediction criterion. Diverse low Reynolds number flow configurations featuring laminar separation bubbles are investigated, such as flat-plate case, NACA 0012 airfoil, SD7003 airfoil and T106C turbine airfoil. The influence of chord-based Reynolds number, angle of attack and inflow turbulence level on the prediction capabilities of the LSTT approach are assessed. Good agreement with available DNS, LES and experimental data is obtained.
\end{abstract}

\section{Nomenclature}
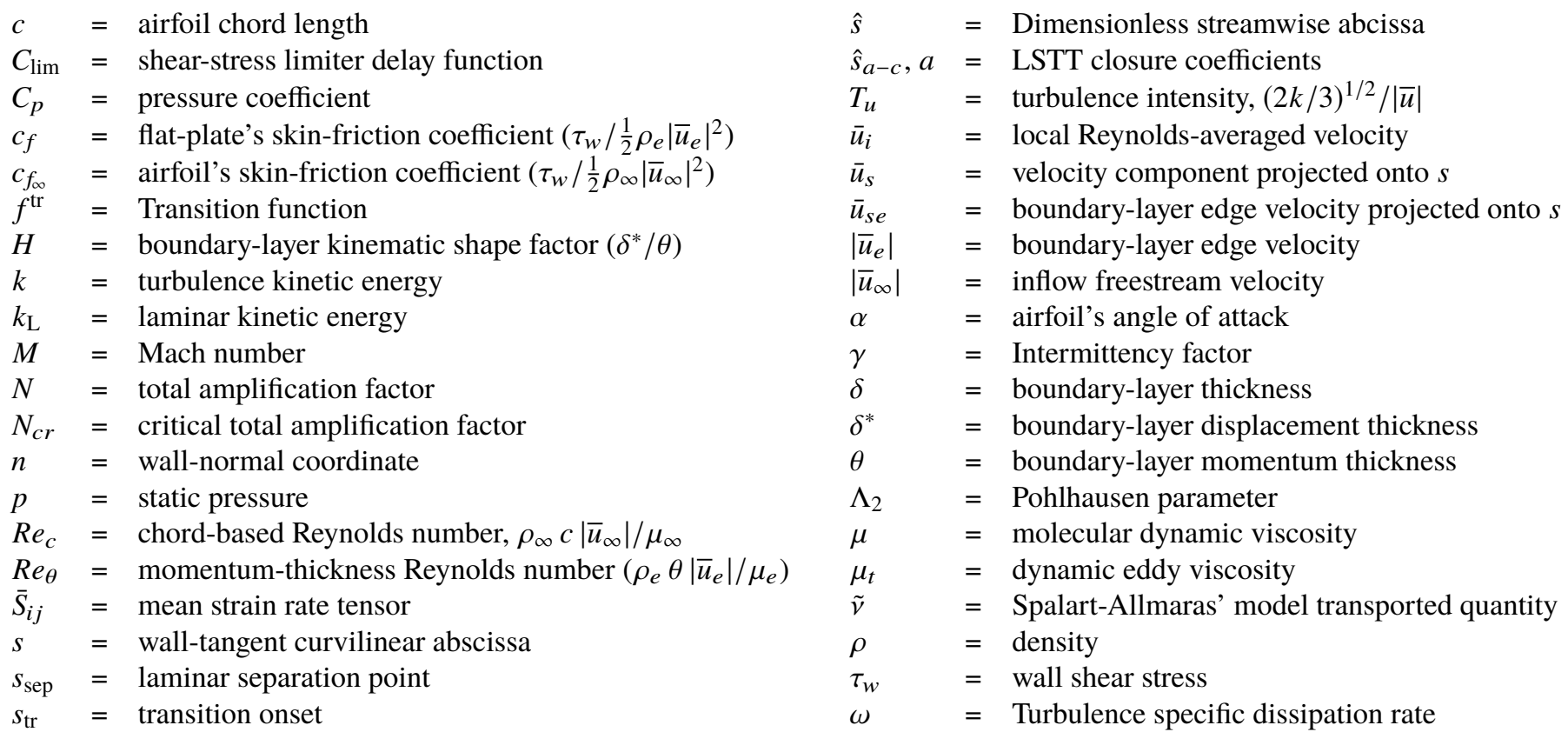

\section{Introduction}

$\mathrm{L}$ AMINAR separation bubbles [1, LSBs] appear in numerous aerodynamic applications, such as dynamic stall

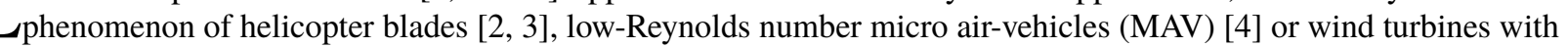
enhanced laminarity [5]. It is well established that the flow initially undergoes laminar separation because of the adverse pressure-gradient [6], followed by transition onset in the separated shear-layer [7], identified as the amplification of Kelvin-Helmholtz instabilities leading to turbulence [8] . Increased turbulent mixing in the shear layer may, depending on flow conditions [9], lead to rapid reattachment (short bubble locally modifying wall-pressure distribution [1]) or

\footnotetext{
* Research Scientist, Aerodynamics, Aeroelasticity and Acoustics Department, Meudon, France. luis.bernardos_barreda@onera.fr

${ }^{\dagger}$ Research Scientist, Aerodynamics, Aeroelasticity and Acoustics Department, Meudon, France. francois.richez@onera.fr

${ }^{\ddagger}$ Research Scientist, Aerodynamics, Aeroelasticity and Acoustics Department, Châtillon, France. vincent.gleize@onera.fr
} 
remain separated for longer distances (long bubble globally modifying wall-pressure distribution [1]). In both cases, airfoil lift, drag and moment are influenced by the presence of the bubble.

RANS (Reynolds-Averaged Navier-Stokes) turbulence models require specific transition information and/or modification to handle the separation-induced transition which dominates the flow [7, 10]. Numerous early [1, 6, 9, 11, 12] and more recent [8,13-15] studies, both experimental and computational, highlight the complex physics of the transitional flow inside LSBs, such as pronounced three-dimensionality, high unsteadiness, turbulent breakdown driven by multiple coherent structures and complex oscillator and amplifier instability mechanisms that may lead to notable changes in the flow topology. Several approaches of varying degree of empiricism have been used to predict this difficult flow.

Ultimately, improved transport-equation based transition models [16] are expected to accurately predict such flows. Correlation-based models [17, 18] reformulate widely used transition correlations [19] into coefficients of transport equations for the intermittency [20, 21] and other transition parameters, to obtain local, and recently [22] Galilean invariant, transition models. Physics-based models [23-25] generally rely on the concept [26] of laminar (pre-transitional) kinetic energy $k_{\mathrm{L}} \approx \frac{1}{2} \overline{u^{\prime 2}}$ (essentially streamwise, in agreement with transition physics [27] and with the observed rapid increase of the Reynolds-stress tensor anisotropy in very low Reynolds number channel flows [28]), which is computed by a specific transport equation, to trigger and control transition in the turbulence model. Transport-equation based transition models are quite successful in mimicking transitional flow effects and in detecting transition [16]. Given the extreme difficulty of accurately predicting transition, algebraic nonlocal transition models are also developed [29], including specific adaptations of additional transport equations [30-32].

Numerous authors have contributed to RANS modeling of LSBs on airfoils, especially at low chord-based Reynolds number $R e_{c}$. Yuan et al. [33] and Windte et al. [34] performed extensive RANS modeling of LSBs for the flow around a SD7003 airfoil at $R e_{c}=60,000$, with various models, and concluded that Menter's BSL [35] $k-\omega$ performed quite well. Source terms were disabled in the laminar regions upstream of transition onset which was determined using an approximate envelope method [34]. Lian and Shyy [36] proposed a nonlocal intermittency model for the activation of Wilcox's $1994 k-\omega$ [37], through direct weighting of the eddy viscosity $v_{t}^{\text {effective }}=v_{t} \gamma$, and determined transition onset by the $e^{N}$ method [38]. Calculations of the SD7003 airfoil at $R e_{c}=60,000$ at various angles of attack gave promising results [36]. The same flow was studied by Catalano and Tognaccini [39] with disabled source terms in the laminar regions upstream of the user-prescribed transition onset. These authors proposed a modification of the $F_{2}$ blending function appearing in the expression of the shear limiter of Menter's $k-\omega$ SST model [35] and of the cut-off value of $\omega$, thus enhancing turbulence production in the transitional region and improving upon the results of the original formulation. An empirical transition onset correlation was added to the method [40] which was applied to various airfoil calculations (SD7003, NACA 0012, S809 and S827). No specific treatment of the transitional region was performed, in the sense that the activation of the turbulence model was made instantly through the source terms at the transition onset.

Despite the encouraging results of the previously described approaches, accurate prediction of LSB is still challenging. As remarked by Dick and Kubacki [16], the quality of the predictions of separation-induced transition remains case-dependent. Numerous existing methods focus on correctly predicting the transition onset, but the transition region prediction still relies on the turbulence model's unapprehended ability to correctly produce turbulence at and downstream of the transition onset. In this paper we present and evaluate a recently developed [41] transition modeling formulation that intends to account for the turbulence growth within a LSB and in the downstream relaxation flow region. To this purpose, laminar separation transition triggering (hereafter LSTT) in the RANS model is progressive in the streamwise direction $(s)$, from the criteria-determined [42-44] transition onset to full turbulence. The assumption is made that the streamwise length between the laminar separation point, which is exactly determined by the solution of the laminar Navier-Stokes equations, and the criteria-determined transition onset is the characteristic lengthscale of the LSTT-process. Therefore, triggering functions of the model are expressed in terms of the aforementioned lengthscale.

Furthermore, wall-normal variations in the LSTT approach are not modeled, in line with many algebraic transition models using intermittency functions [36, 45]. The coefficients that define the algebraic form of the triggering functions control the production and destruction of turbulent quantities. They are calibrated to obtain the best possible agreement with available LSB DNS data [46, 47].

Although most methods dealing with the transition region use the concept of intermittency [21, 22], the transitiontriggering functions used in the present work are not directly related to this concept, but should rather be considered as "empirical weighting coefficients" [48] designed to reproduce as best as possible the progressive growth of turbulence in LSBs. Therefore they are not necessarily bounded by 1 , but may be designed to locally overshoot this bound.

In this work we use linear $k$ - $\omega$ models [49, p.124] which are largely used as the underlying RANS framework of transition models [37, 50]. Additionally, we extend the LSTT approach to the one-equation Spalart-Allmaras [51] turbulence model. 
This paper is organized as follows. In $\$[1]$ we present the turbulence models used, the transition onset prediction models, and the turbulence triggering approaches. In $\$[\mathrm{IV}$, the models are evaluated for the prediction of the flow around a NACA 0012 airfoil, SD7003 airfoil and T106C turbine blade.

\section{Methodology description}

\section{A. Turbulence models}

In this work we use two different turbulence models, the $k-\omega$ Wilcox 2006 [52] model and the one-equation SpalartAllmaras turbulence model [51]. Transitional adaptations are performed on the original fully-turbulent formulation of both models. The LSTT approach is implemented in each model through introduction of the dimensionless transition function $f^{\text {tr }}$ in the source terms and the dimensionless stress limiter delay function $C_{\text {lim }}$.

The set of equations of the $k-\omega$ model is summarized as follows,

$$
\begin{aligned}
& \partial_{t}(\rho k)+\partial_{i}\left(\rho \bar{u}_{i} k\right)= f^{\operatorname{tr}}\left(-\rho \overline{u_{i}^{\prime} u_{j}^{\prime}}\right)\left(\partial_{j} \bar{u}_{i}\right)-\beta_{k} \rho \omega k+\partial_{i}\left[\left(\mu+\sigma_{k} \mu_{t}\right) \partial_{i} k\right] \\
& \partial_{t}(\rho \omega)+\partial_{i}\left(\rho \bar{u}_{i} \omega\right)= \min \left(f^{\mathrm{tr}}, 1\right) \alpha_{\omega} \frac{\omega}{k}\left(-\rho \overline{u_{i}^{\prime} u_{j}^{\prime}}\right)\left(\partial_{j} \bar{u}_{i}\right)-\beta_{\omega} \rho \omega^{2}+\partial_{i}\left[\left(\mu+\sigma_{\omega} \mu_{t}\right) \partial_{i} \omega\right] \\
&+\sigma_{d} \frac{\rho}{\omega} \max \left(\left(\partial_{i} k\right)\left(\partial_{i} \omega\right), 0\right) \\
&\left(-\rho \overline{u_{i}^{\prime} u_{j}^{\prime}}\right) \approx 2 \mu_{t} \bar{S}_{i j}-\frac{2}{3} \rho k \delta_{i j} \\
& \mu_{t}= \frac{\rho k}{\max \left\{\omega ; C_{\lim } \sqrt{\frac{2 \bar{S}_{i j} \bar{S}_{i j}}{\beta_{k}}}\right\}}
\end{aligned}
$$

Through Eqns. (1a) and (1b) one may observe that the transition function $f^{\text {tr }}$ acts on the production terms of both $k$ and $\omega$. As will be presented in the following paragraphs, the purpose of the $f^{\text {tr }}$ function is to weight the modeled effective production of turbulence at and downstream of the transition onset, in order to account for the relatively high Reynolds shear stresses identified experimentally in the transitional region. In order to allow for this important production of turbulence, the weighting factor of the production of $\omega$ is limited to 1 . This is necessary because otherwise the boost of turbulence production expected by the use of $f^{\text {tr }}>1$ via the production term of $k$ would be mitigated by a boost in the production of the $\omega$ equation.

The purpose of the $C_{\text {lim }}$ function, which is a constant in the original Wilcox' 2006 model, is to delay the activation of the shear stress limiter, in order to allow for sufficiently high production rates of turbulence in the transitional region. As it can be anticipated, $C_{\lim }=0$ in the transitional region, thus disabling the shear stress limiter and allowing for important turbulence production, and it yields $C_{\text {lim }}=7 / 8$ in the fully turbulent region.

The Spalart-Allmaras [51] model adapted to the LSTT formulation, without tripping term, may be summarized as follows,

$$
\begin{aligned}
\partial_{t}(\tilde{v})+\partial_{i}\left(\bar{u}_{i} \tilde{v}\right)= & f^{\mathrm{tr}} c_{b 1}\left(1-f_{t 2}\right) \tilde{S} \tilde{v}-\min \left(f^{\mathrm{tr}}, 1\right)\left(c_{w 1} f_{w}-\frac{c_{b 1}}{\kappa^{2}} f_{t 2}\right)\left(\frac{\tilde{v}}{n}\right)^{2} \\
& +\frac{1}{\sigma}\left[\partial_{i}\left((v+\tilde{v}) \partial_{i} \tilde{v}\right)+c_{b 2}\left(\partial_{i} \tilde{v}\right)^{2}\right] \\
\left(-\overline{u_{i}^{\prime} u_{j}^{\prime}}\right)= & 2 v_{t} \bar{S}_{i j} \\
v_{t}= & \tilde{v} f_{v 1}
\end{aligned}
$$

Through the Eqn. 22a) one may observe that the $f^{\text {tr }}$ function acts on both the production and destruction terms of $\tilde{v}$. We limit the weighting factor of the destruction of $\tilde{v}$ to 1 . This is done for the same reason as the production of $\omega$ is limited in the $k-\omega$ formulation, i.e. to allow high rate of turbulence growth in the transitional region where $f^{\text {tr }}>1$. As one may observe through the equations that constitute the Reynolds shear stresses [Eqns. (2b) and (2c)] it has not been needed to implement the $C_{\text {lim }}$ function in the Spalart-Allmaras model using the LSTT approach. 


\section{B. Transition onset prediction}

Before examining the formulation of the functions $f^{\text {tr }}$ and $C_{\text {lim }}$, we note that the LSTT approach requires information on the transition onset location, namely $s_{\mathrm{tr}}$. For this purpose, we employ the Arnal-Habiballah-Delcourt [43, 53, 54] (AHD) and the Gleyzes [44] criteria for the natural attached and separated transition onset computation of the boundarylayer. These criteria are computed at each iteration step of the simulation, and provide the $s_{\text {tr }}$ point used by the $f^{\text {tr }}$ and $C_{\lim }$ functions. The AHD-Gleyzes transition criteria, which are based on linear stability analysis and experimental correlations, are employed to model the amplification of Tollmien-Schlichting and Kelvin-Helmholtz waves. For this reason, these criteria are best suited to natural transition prediction, i.e. at low external turbulence level conditions. The criteria evaluate non-local algebraic expressions employing boundary-layer quantities along the streamwise coordinate $s$.

The incompressible formulation of the AHD criterion is expressed as follows,

$$
R e_{\theta \mathrm{tr}}=-206 \exp \left(25.7 \overline{\Lambda_{2}}\right)\left[\ln \left(16.8 T_{u_{\mathrm{tr}}}\right)-2.77 \overline{\Lambda_{2}}\right]+R e_{\theta \mathrm{cr}}
$$

The transition onset is located at the point $s=s_{\mathrm{tr}}$ where $R e_{\theta(s)}=R e_{\theta \mathrm{tr}}$ is reached. The threshold of transition is determined by the value of the transitional external turbulence level, $T_{u_{\mathrm{tr}}}$. The subscript "cr" denotes the laminar critical point, which is located at $s=s_{\mathrm{cr}}<s_{\mathrm{tr}}$ when $\operatorname{Re}_{\theta(s)}=R e_{\theta c r}=\exp \left(52 / H_{(s)}-14.8\right)$. The mean Pohlhausen parameter $\overline{\Lambda_{2}}$ is expressed as follows,

$$
\overline{\Lambda_{2}}=\frac{1}{s-s_{\mathrm{cr}}} \int_{s_{\mathrm{cr}}}^{s} \frac{-\theta^{2}}{\mu\left|\bar{u}_{e}\right|} \frac{\partial p_{\text {wall }}}{\partial s} \mathrm{~d} s
$$

Where $\left|\bar{u}_{e}\right|$ denotes the magnitude of the velocity at the boundary-layer edge and $p_{\text {wall }}$ the pressure at the wall.

The AHD criterion is used from the laminar critical point up to the point where $H=2.8$. If the transition onset has not been reached at such point, named the switch point, then the transition onset prediction is relayed by the Gleyzes criterion, which models the total amplification factor and is expressed as follows,

$$
N(s)=N_{\mathrm{sw}}+\int_{\operatorname{Re}_{\theta \mathrm{sw}}}^{R e_{\theta}} \frac{-2.4}{\mathcal{B}_{G L}} \mathrm{~d} R e_{\theta}
$$

Where $\mathcal{B}_{G L}$ is the Gleyzes function and is expressed as follows,

$$
\mathcal{B}_{G L}=\left\{\begin{array}{rll}
\frac{-162.11093}{H^{1.1}} & \text { for } & 3.36<H \\
-73[\exp [-1.56486(H-3.02)]] & \text { for } & 2.8<H \leq 3.36 \\
-103[\exp [-4.12633(H-2.8)]] & & \text { else }
\end{array}\right.
$$

The transition onset is located at the critical total amplification factor $N_{\mathrm{cr}}=N\left(s=s_{\mathrm{tr}}\right)$ of Eqn. (5). This critical value is related to the threshold transitional external turbulence level $T_{u_{\mathrm{tr}}}$ via the Mack's [55] relationship Eqn. (7),

$$
N_{c r}=-2.4 \ln \left(T_{u}\right)-8.43
$$

The switch total amplification factor $N_{\mathrm{sw}}$ expressed in Eqn. (5) accounts for the laminar boundary-layer receptivity history up to the switch point. It is determined by replacing $\operatorname{Re}_{\theta \text { tr }}$ by current $\operatorname{Re}_{\theta}\left(s=s_{\mathrm{sw}}\right)$ of Eqn. (3); inject the Mack's relationship Eqn. (7) into Eqn. (3), and finally solving for $N\left(s=s_{s w}\right)$,

$$
N_{\mathrm{sw}}=-8.43-2.4 \ln \left[\frac{1}{16.8} \exp \left(2.77 \overline{\Lambda_{2}}-\frac{R e_{\theta}-R e_{\theta \mathrm{cr}}}{206 \exp \left(25.7 \overline{\Lambda_{2}}\right)}\right)\right]
$$

From the author's experience in using the Gleyzes criterion, we discourage the use of actual boundary-layer values in the neighborhood of a deeply detached boundary-layer. The reason is that massively detached boundary-layers may yield a local decrease of $\operatorname{Re}_{\theta}(s)$ before the transition onset is reached, thus compromising the growth of $N$ via Eqn. (5). This would provoke a permanent downstream displacement of the transition onset which would translate into numerical instabilities and poor prediction precision. In order to solve this issue, we use an extrapolation approach of $H$ and $\operatorname{Re}_{\theta}$ values, from a set of points located in the neighborhood of the laminar separation point (cf appendix $\sqrt{\mathrm{A}}$ ).

Further details on the implementation of the criteria-based approach are presented by Cliquet et al. [45] and Perraud et al. [56]. 


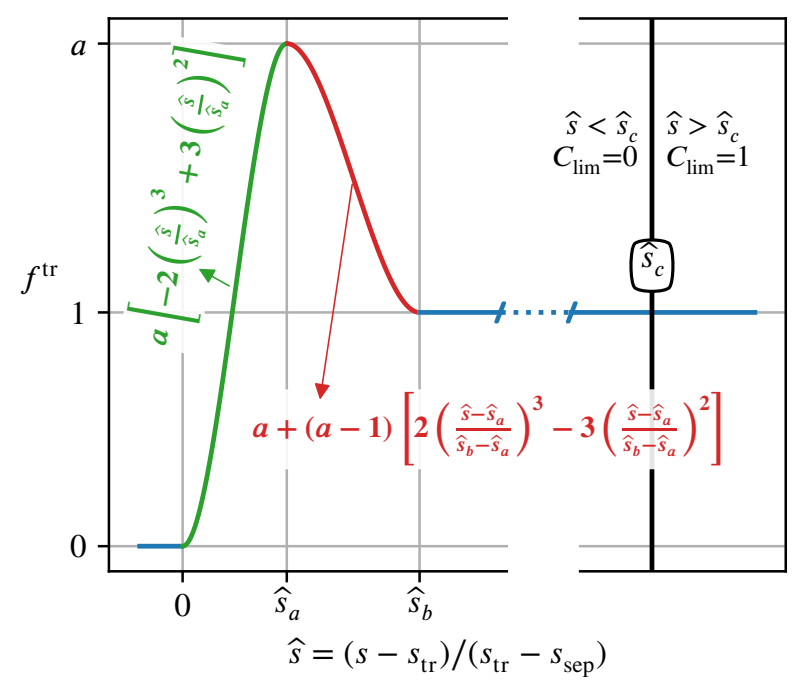

Fig. 1 Scheme of the transition function $f^{\text {tr }}\left(\mathbf{C}^{1}\right.$ piecewise cubic polynomials) and shear stress limiter switch $C_{\lim }$ used in the LSTT [41] approach with the closure constants $\widehat{s}_{a}, \widehat{s}_{b}, \widehat{s}_{c}$ and $a$ adapted to the turbulence model as shown in Table 1 .

\section{Turbulence triggering modeling and calibration}

In this section we present the algebraic functions $f^{\text {tr }}$ and $C_{\text {lim }}$ used for triggering the turbulence models [Eqns. (1) and [2] ] at and downstream of the transition onset $s_{\mathrm{tr}}$. As these functions are applied everywhere in the flowfield, they yield specific values at every point of the flow, i.e. $f^{\mathrm{tr}}(x, y, z)$ and $C_{\lim }(x, y, z)$. In order to determine these values throughout the entire flowfield, a two-step procedure is performed as follows.

In a first step, only the values of $f^{\text {tr }}$ and $C_{\lim }$ at the wall ought to be determined. This is accomplished by specific algebraic dependence of $f^{\mathrm{tr}}$ and $C_{\lim }$ upon $s$, the curvilinear abscissa of the wall, as shown in Fig. 1 In practice, for the airfoil simulations of the current work, $s$ corresponds to the airfoil's wall contour.

For the determination of $f^{\mathrm{tr}}(s)$ and $C_{\lim }(s)$, the LSTT model makes use of the transition onset location, $s_{\mathrm{tr}}$, provided by the transition criteria presented in $\$ \mathrm{~B}$, and the laminar separation point $s_{\mathrm{sep}}$, retrieved by direct prediction of the Navier-Stokes equations. Hence, the dimensionless curvilinear abscissa $\widehat{s}$ is constructed as presented in Eqn. 99,

$$
\widehat{s}:=\frac{s-s_{\text {tr }}}{s_{\text {tr }}-s_{\text {sep }}}
$$

Once the wall values $f^{\operatorname{tr}}(s)$ and $C_{\mathrm{lim}}(s)$ are determined, in a second step we perform a wall-normal extrusion of their values up to the boundary-layer height, namely $\delta$. Outside the boundary-layer, the constant values $f^{\text {tr }}=1$ and $C_{\text {lim }}=1$ are set. In this way, $f^{\text {tr }}$ and $C_{\lim }$ are properly defined at every point in space.

The main role of the LSTT model is to weight the rate at which turbulence is produced from the transition onset. The reason is to account for the smooth and relatively important production of the Reynolds shear stress, $-\overline{u^{\prime} v^{\prime}}$, that occurs in the separated shear layer close to the transitional region, as observed in experiments [8, 57] and DNS [47]. This is particularly required in order to avoid the typically low values of skin-friction RANS predictions downstream of the bubble [58]. Proper growth of the Reynolds shear stress is accomplished by boosting the triggering function $\left(f^{\text {tr }}>1\right)$, which facilitates a strong imbalance of production-to-dissipation ratio of turbulence in the transitional region. The $k-\omega$ model additionally requires a delay on the use of the shear stress limiter up to the region where an established turbulent boundary-layer is expected. This location, governed by the $\widehat{s}_{c}$ closure coefficient, is situated several bubble lengths downstream of the bubble.

As observed in Fig 1 , the triggering region scales with the bubble's laminar length, $s_{\mathrm{tr}}-s_{\mathrm{sep}}$, thanks to the use of the dimensionless abscissa coordinate formulation of Eqn. (9). This feature is convenient in order to automatically adapt the turbulence triggering to different bubble sizes.

In this work we also present results of a simple off-on triggering of the turbulence model. This commonly used approach, that we denote here as 'Step', consists in disabling the source terms of the turbulence model upstream of the transition onset location. The Step approach is presented with the shear stress limiter enabled. 

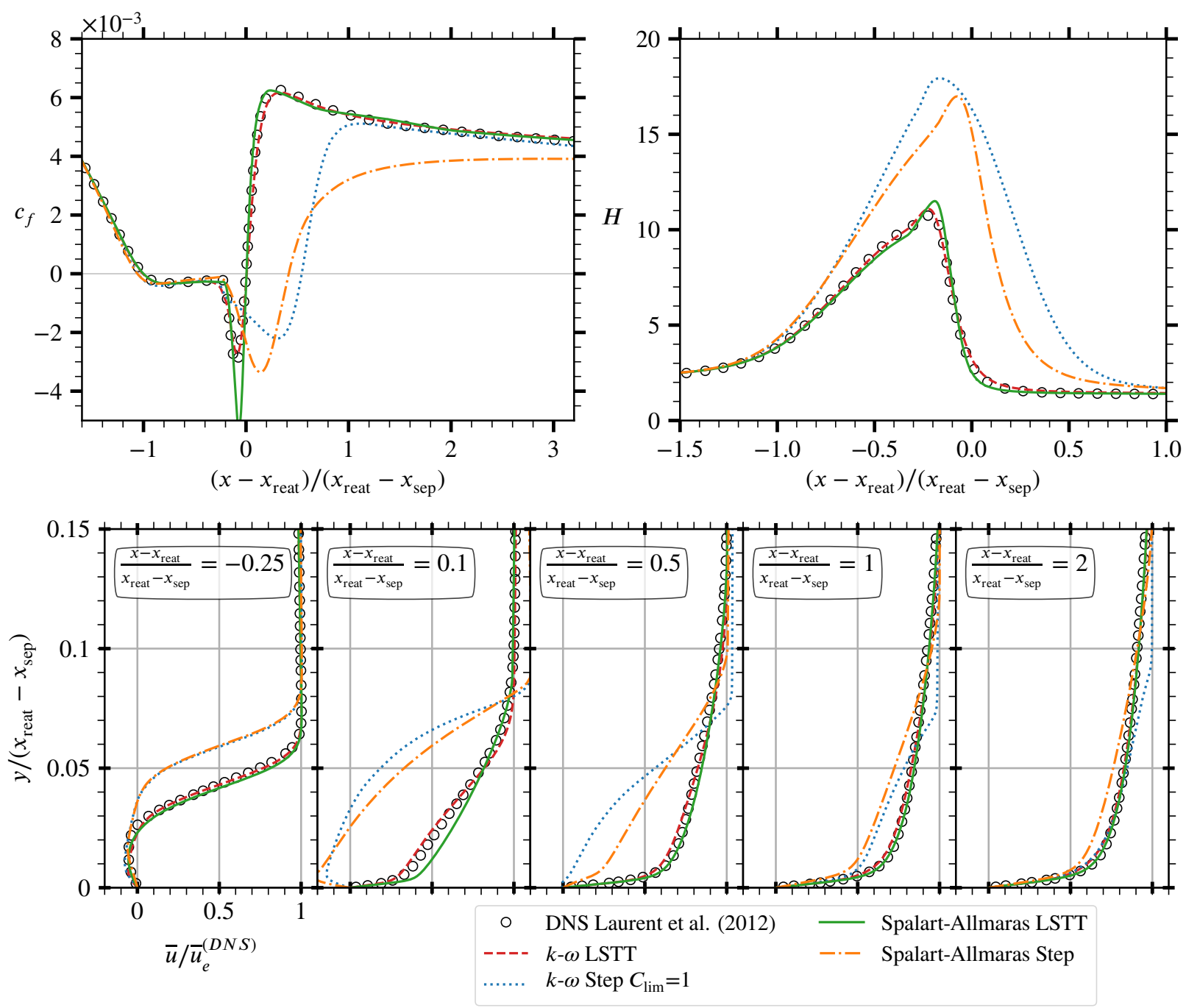

Fig. 2 Comparison of predicted skin-friction, shape factor and velocity profiles using the Step and the LSTT approach, coupled with the $k-\omega[52]$ and Spalart-Allmaras [51] turbulence models, against the DNS data of Laurent et al. [47] over a flat-plate configuration with adverse pressure gradient. 
The closure constants of the LSTT model $\left(\widehat{s}_{a}, \widehat{s}_{b}, \widehat{s}_{c}\right.$ and $\left.a\right)$ were calibrated [41] to reproduce the $c_{f}$ distribution of the bubble of a flat-plate DNS reference [47] using $k-\omega$ models. In Table 1 we also present the calibration adapted to the Spalart-Allmaras turbulence model, as shown in Fig. 2. As it can be observed, the LSTT calibration for both models is in correct agreement with the DNS skin-friction. Only a slight overestimation of the minimum value of $c_{f}$ is identified for the Spalart-Allmaras model. An interesting observation of Fig. 2 is that not only the $c_{f}$ predictions of the LSTT model are in better agreement with DNS, but also the shape factor $H$ and the velocity profiles.

\begin{tabular}{rcccc}
\hline Turbulence model & $\widehat{s_{a}}$ & $\widehat{s_{b}}$ & $\widehat{s_{c}}$ & $a$ \\
\hline \hline$k-\omega$ Wilcox 2006 [52] & 0.182 & 0.414 & 5.83 & 2.15 \\
Spalart-Allmaras [51] & 0.482 & 3.107 & - & 7.03 \\
\hline
\end{tabular}

Table 1 LSTT [41] closure coefficients for the considered turbulence models

\section{Results}

In the following paragraphs, the LSTT approach is evaluated using the established calibration of Table 1

\section{A. NACA 0012 Airfoil}

In this section we present an assessment of the LSTT approach using $k-\omega$ Wilcox (2006) and Spalart-Allmaras turbulence models for two different operating conditions of the NACA 0012 airfoil. These conditions were chosen because high-fidelity simulations are available in literature. The first operating condition comes from the DNS of Jones et al. [13], which yields $\alpha=5^{\circ}$ and $R e_{c}=50,000$. The second condition is issued from the ILES of Alferez et al. [59], which yields $\alpha=10.55^{\circ}$ and $R e_{c}=100,000$.

DNS Jones et al. [13] with forced perturbations DNS Jones et al. [13] unforced

LSTT $N_{c r}=6\left(T_{u} \approx 0.245 \%\right)$

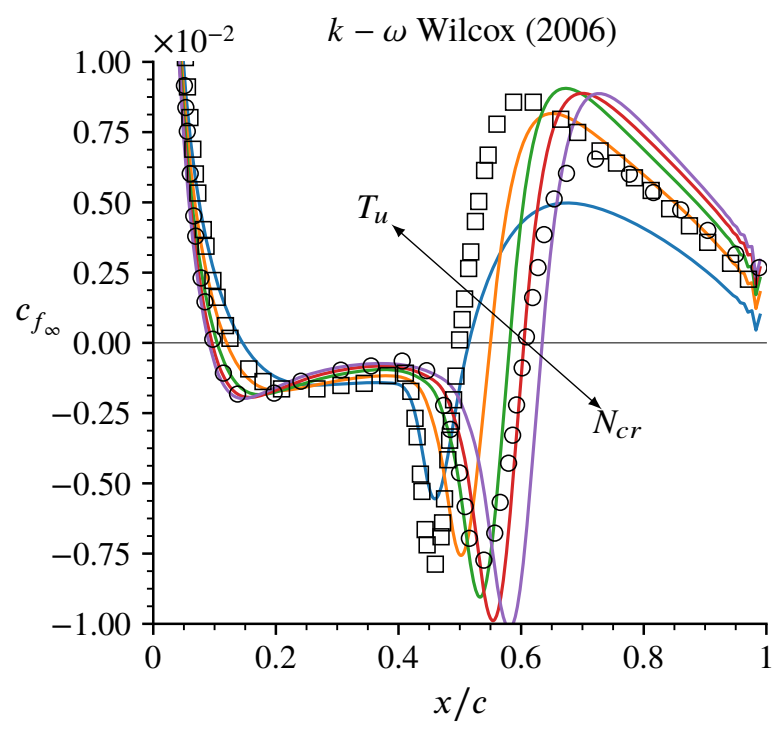

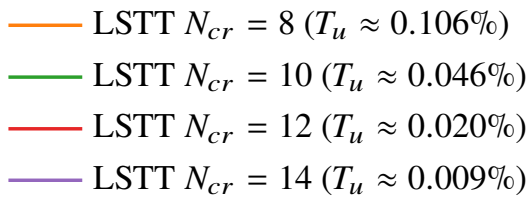

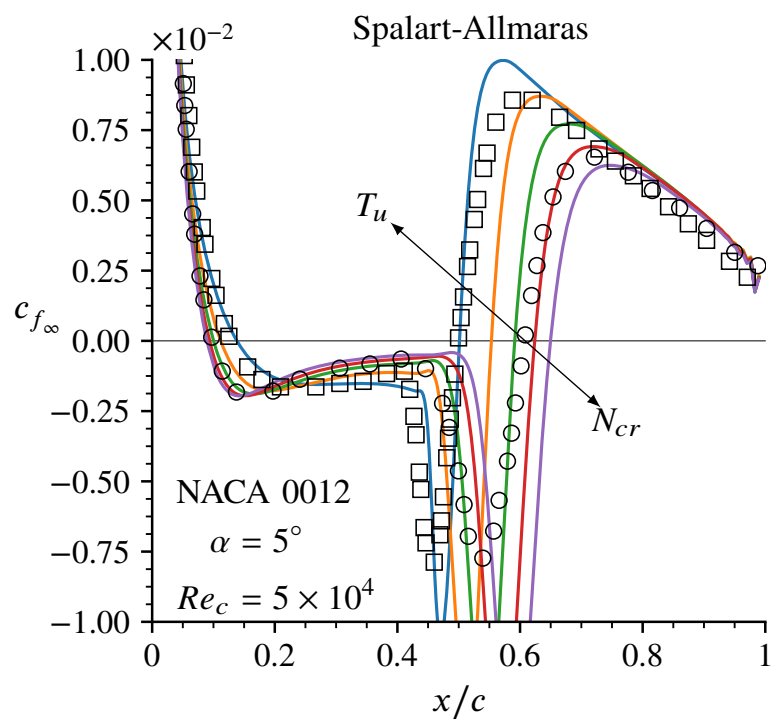

Fig. 3 Skin-friction RANS predictions of the influence of the transitional turbulence level threshold, $T_{u}$ (or $N_{c r}$ ), on the suction side of a NACA 0012 at $\alpha=5^{\circ}$ and $R e_{c}=50,000$ using $k-\omega$ Wilcox (2006) [52] and Spalart-Allmaras [51] turbulence models with AHD-Gleyzes [44, 53] transition onset prediction criteria and LSTT [41] turbulence activation against DNS data of Jones et al. [13]. 
In Fig. 3 the RANS predictions of skin-friction using LSTT are compared against the DNS data of Jones et al. [13]. Two different DNS data sets are presented, resulting from a forced and unforced strategies. The forced DNS performs a velocity perturbation with $0.1 \%$ of amplitude of the free-stream velocity at the location $(x / c, y / c)=(0.1,0.129)$ yielding several frequencies that optimally excite the transition to turbulence within the separated shear-layer [13]. The unforced DNS yields a self-sustained transition [13], which is also observed in many unforced high-fidelity simulations [59-62].

The RANS computations presented in Fig. 3 were performed using automatic transition prediction via AHD-Gleyzes [44, 53] criteria. A sweep of the $N_{c r}$ parameter (which is related to the turbulence intensity via Mack's relationship [55]) was performed between $N_{c r}=6$ and $N_{c r}=14$. The influence of turbulence level upon the displacement of separation and reattachment points is well captured by both turbulence models using LSTT. Indeed, Fig. 3 shows that an increase in turbulence level produces a downstream displacement of the separation point and an upstream displacement of the reattachment point. The RANS predictions using both turbulence models in Fig. 3 show that $N_{c r}=12$ curves match quite well the unforced DNS data. Furthermore, RANS predictions at $N_{c r}=6$ agree quite well with forced DNS data.

However, it is difficult to evaluate the precision of the transition onset prediction criteria based upon the DNS data. The forced DNS data yield a forced perturbation of $0.1 \%$, which could be assimilated to $N_{c r} \approx 8$ following Mack's relationship. For $N_{c r}=8$, the RANS simulations predict a bubble which is slightly longer than the forced DNS data. This would mean that the transition criterion tends to predict a slightly downstream transition onset. However, the assumption that $N_{c r}=8$ shall be privileged because DNS was forced with a perturbation of $0.1 \%$ of amplitude may be overestimated. Nonetheless, from the results of Fig. 3 a,d the available information on the perturbation forcing [13], we may infer that transition onset prediction is accurate within an uncertainty of about $\Delta N_{c r} \approx 2$, which we consider quite successful for this case.

In the following we present a comparison of RANS computations against the ILES of Alferez et al. [59] for the NACA 0012 at $\alpha=10.55^{\circ}$ and $R e_{c}=10^{5}$. In this case, we compare the predictions of both turbulence models using LSTT and typical Step off-on activation, as well as the predictions using Menter-Langtry $\gamma-\tilde{R} e_{\theta t}$ model.

Typical boundary-layer parameters are shown in Fig. 4. The skin-friction and pressure coefficients are computed as $c_{f_{\infty}}=\tau_{w} /\left(0.5 \rho_{\infty}|\bar{u}|_{\infty}^{2}\right)$ and $C_{p}=\left(p-p_{\infty}\right) /\left(0.5 \rho_{\infty}|\bar{u}|_{\infty}^{2}\right)$, respectively. As the ILES [59] was unforced, no accurate physics-based information is available on the most appropriate value of $N_{c r}$ to be used. Therefore, preliminary sweeps on the value of $N_{c r}$ were performed (similarly to those presented in Fig. 33) that allowed us to infer that $N_{c r}=12$ is an acceptable choice. Note that $N_{c r}=12$ yielded also good RANS predictions for the unforced DNS of Jones et al. [13] shown in Fig. 3

We can observe in Fig. 4 that the computations of $\gamma-\tilde{R} e_{\theta t}$ and Step activations of $k-\omega$ and Spalart-Allmaras turbulence models produce a stalled condition. This effect can be seen through the skin-friction evolution. For the three aforementioned RANS computations, once the laminar boundary layer separates at about $x / c \approx 0.01$, it remains separated $\left(c_{f_{\infty}}<0\right)$ up to the trailing edge. The stalled condition predicted by these models can also be seen through the pressure distribution (where they present a lack of suction near the leading edge), the momentum thickness Reynolds number (where they present a too pronounced slope) and the boundary-layer shape factor (where they present very high values all over the airfoil, showing a massive separation). On the contrary, the LSTT activation of the RANS models produce more accurate predictions with respect to the ILES data. The bubble topology is well predicted by the LSTT-activated models, as the reattachment points are very close to the ILES and the characteristic positive peaks of skin-friction right downstream of the bubble are well captured. The Spalart-Allmaras LSTT activation produces a too pronounced negative peak of skin-friction, as it is systematically observed (Figs. 3 and 2). Furthermore, the LSTT-predicted evolution of $R e_{\theta}$ and $H$ follows the abrupt jumps in the transitional region $(0.05 \lesssim x / c \lesssim 0.1)$ that are characteristic of LSBs. Nevertheless, the pressure coefficient suction peak on the bubble region is slightly overestimated by the LSTT predictions.

The velocity profiles predicted by the different RANS computations can be observed in Fig. 5. In line with the boundary-layer predictions presented in the previous paragraph, Fig. 5 shows that $\gamma-\tilde{R} e_{\theta t}$ and Step-activation computations predict a stalled condition. The laminar separation $(x / c=0.05)$ is properly predicted by the aforementioned models, but from $x / c \gtrsim 0.075$ the velocity profiles begin to deviate from the ILES data, probably due to an insufficient production of turbulence stress. On the contrary, the LSTT-activated RANS models accurately follow the ILES evolution in the separated laminar region $(x / c=0.05)$, the transitional $(x / c=0.075)$ and reattachment $(x / c=0.1)$ regions, and even further downstream. From about $x / c \approx 0.5$ the LSTT models predict a more filled velocity profile than the ILES. This is coherent with the slight underestimation of $H$ observed in Fig. 4f from about $x / c \gtrsim 0.5$. 

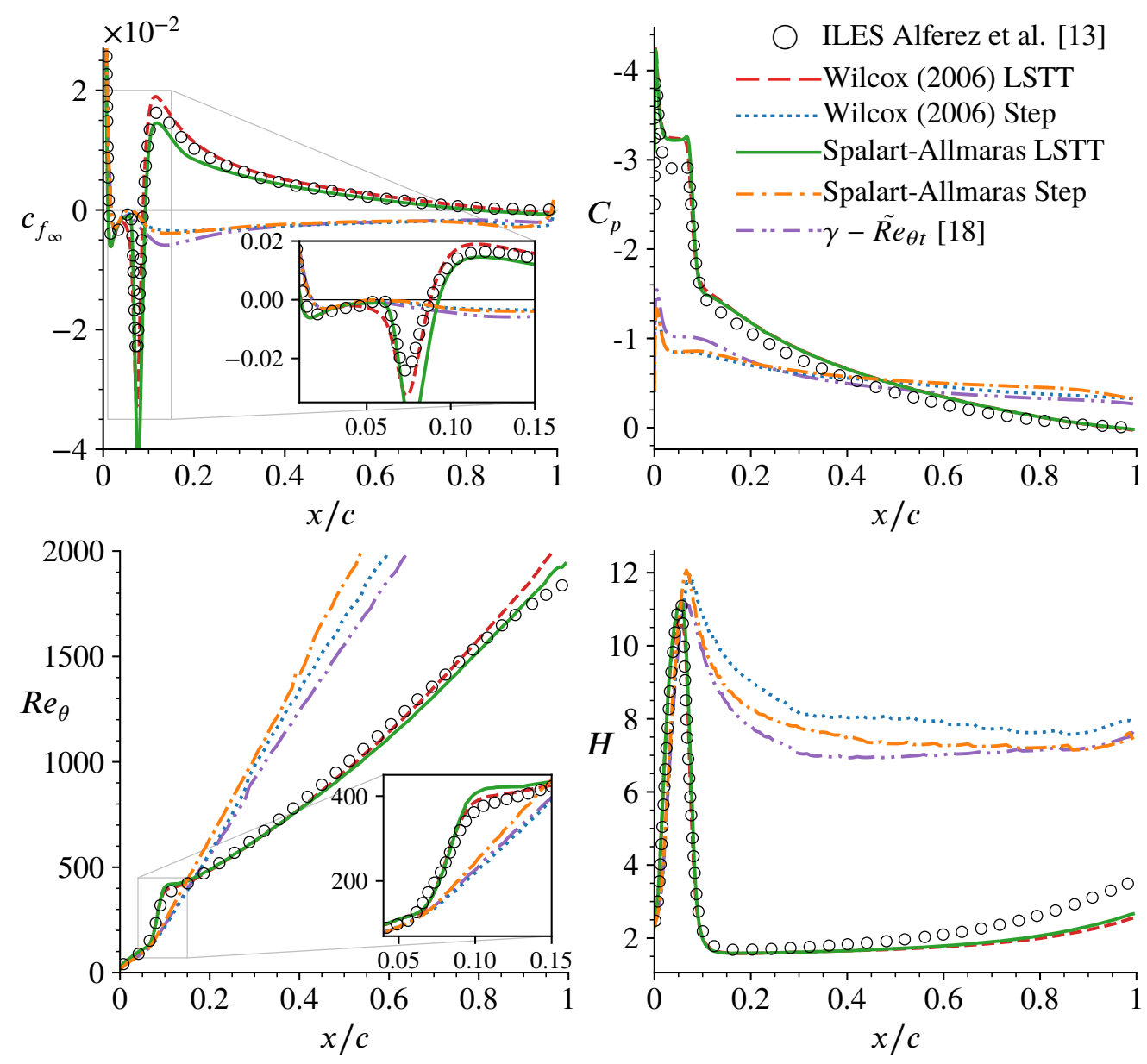

Fig. 4 Boundary-layer parameters on the suction side of the NACA 0012 airfoil at $\alpha=10.55^{\circ}$ and $R e_{c}=10^{5}$ predicted by several RANS models compared against ILES results [59].

\section{B. SD7003 Airfoil}

In this section we present the results of the RANS predictions on the flow around the SD7003 [63] airfoil. Several flow conditions are analyzed, as well as different prediction approaches.

In Fig. 6 we compare RANS predictions with available unforced LES data [60, 64] at $\alpha=4^{\circ}$ and $R e_{c}=60,000$. Several RANS approaches were employed. The LSTT activation was done using automatic transition prediction criteria [43, 44] using $N_{c r}=11$. The choice of $N_{c r}=11$ was done following a previous (not shown here) sweep on $N_{c r}$ (similarly to the sweep shown in Fig. 3) and by choosing the value that yielded the closest prediction with respect to the LES data. Remarkably, the chosen value for the present case, $N_{c r}=11$, is very close to $N_{c r}=12$, which was the value that produced the best LSTT predictions of the transition onset of the flows computed using unforced DNS [13] and unforced ILES [59] of Figs. 3 and 4, respectively. The Step-activated RANS simulations used in the current study were performed by imposing the activation location exactly at the transition onset location predicted by the LSTT model, which is judged to be accurate enough.

As we observe in Fig. 6, the Menter-Langtry $\gamma-\tilde{R e} e_{\theta t}$ model (used here with a threshold of $N_{c r}=11$ as well) and the Step-activated Spalart-Allmaras model produce very close predictions. The $\gamma-\tilde{R} e_{\theta t}$ model properly predicts the transition onset location, based on two observations: (1) the predicted minimum skin-friction peak is located close to the minimum peak of skin-friction of the LES at $x / c \approx 0.6$; and (2) the end of the $C_{p}$ plateau agrees reasonably well with the LES one.

However, downstream of the transition onset, the $\gamma-\tilde{R} e_{\theta t}$ model predicts a reattachment point that is located $13 \%$ of the chord downstream of the LES reattachment point. The skin-friction downstream of the bubble predicted by $\gamma-\tilde{R} e_{\theta t}$ model is underestimated by a factor of $\approx 3$. These observations downstream of the transition onset are related 


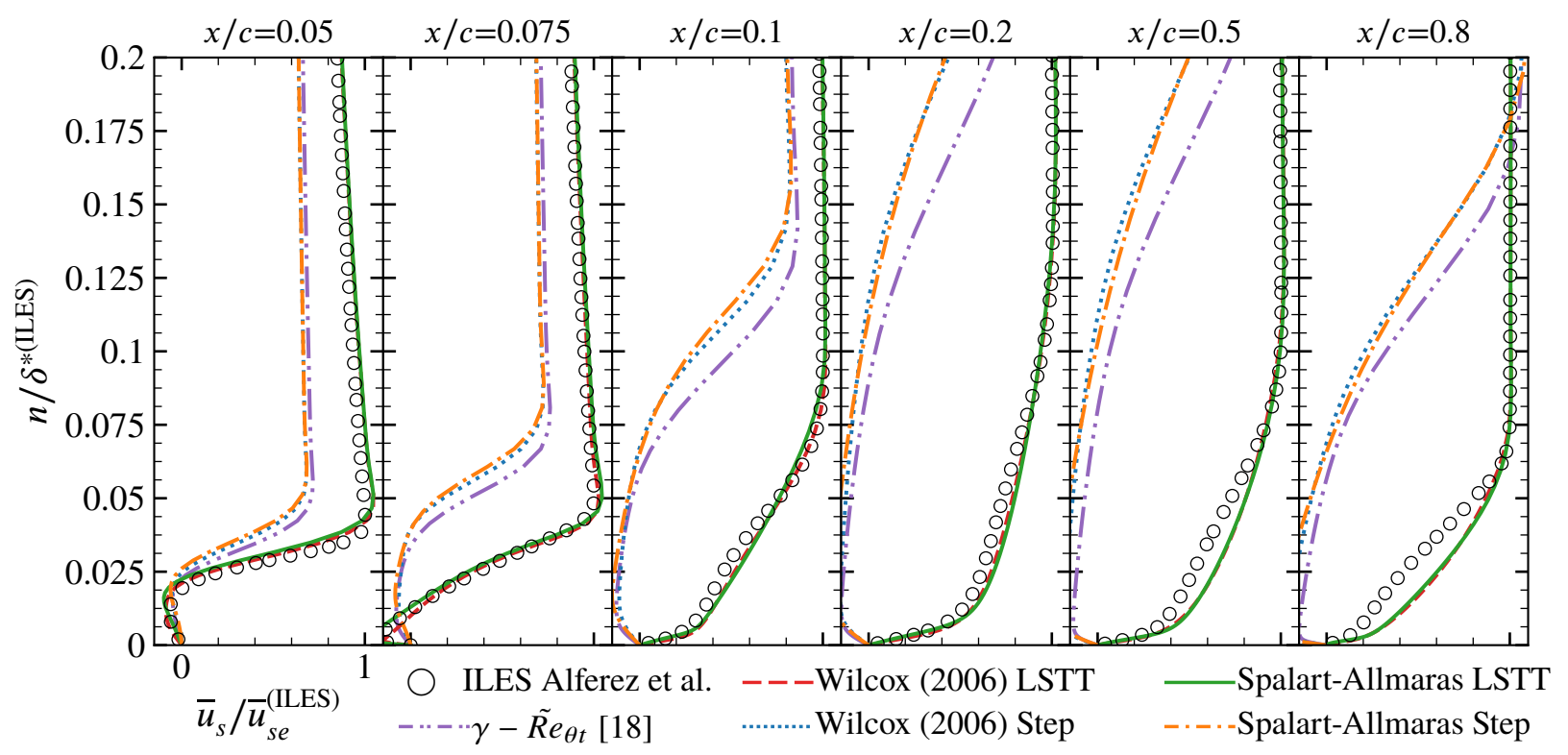

Fig. 5 Comparison of velocity profiles for several models for the NACA 0012 at $\alpha=10.55^{\circ}$ and $\operatorname{Re}_{c}=10^{5}$ [59]. The airfoil-tangential velocity $\bar{u}_{s}$ is plotted against the airfoil's wall-normal direction $n$. The tangential velocity component has been scaled by its value at the boundary layer edge of the ILES data $\bar{u}_{s e}^{(\mathrm{ILES})}$. The airfoil's distance to the wall $n$ has been scaled with respect to the integral displacement thickness of the ILES data $\delta^{* \text { (ILES) }}$.

ILES Galbraith and Visbal [64]

$\triangle$ LES Catalano and Tognaccini [60]

$---k-\omega$ Wilcox (2006) LSTT

$k-\omega$ Wilcox (2006) Step

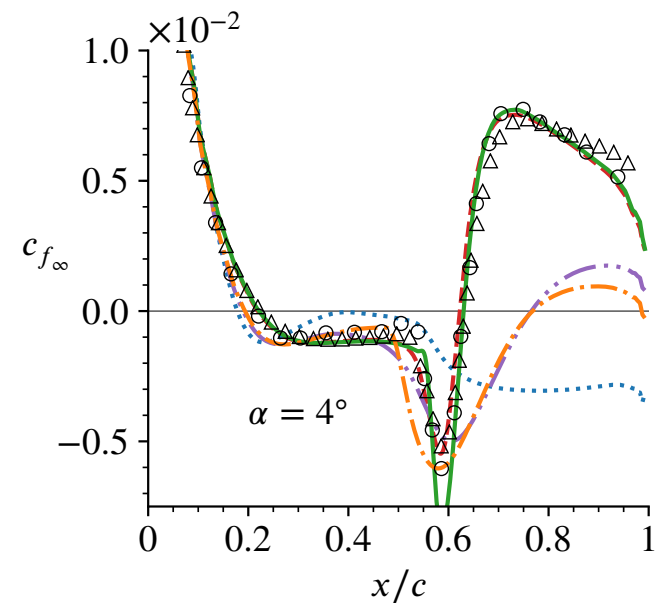

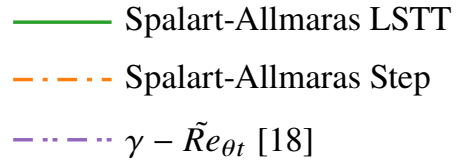

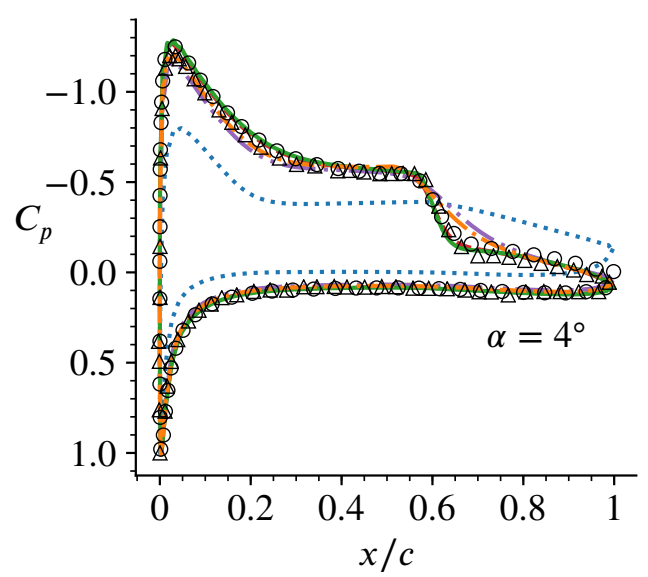

Fig. 6 Comparison of predicted skin-friction (on the suction side) and pressure coefficient using several RANS approaches against the LES data of Catalano and Tognaccini [60] and ILES data of Galbraith and Visbal [64] of the flow around a SD7003 airfoil $\left(M_{\infty}=\mathbf{0 . 1 6}, \alpha=4^{\circ}, R e_{c}=\mathbf{6 0 , 0 0 0}\right)$ predicted using $N_{c r}=11$.

to the slow recovery of $C_{p}$ predicted by $\gamma-\tilde{R} e_{\theta t}$ in the interval $0.6 \lesssim x / c \lesssim 0.8$, where it can be observed that the $\gamma-\tilde{R} e_{\theta t}$ curve of $C_{p}$ is not sufficiently steep. Analogous observations can be done with respect to the Spalart-Allmaras model with Step activation, viz. the bubble length is overestimated, the skin-friction values downstream of the bubble are underestimated, and the recovery of pressure downstream of the transition onset is too slow. The Step-activated $k-\omega$ model predicts an open-bubble topology, which is likely due to the slow turbulence production in the transitional 
region provoked by both the Step-activation and the use of the shear stress limiter (1d).

On the other hand, the LSTT activations using both $k-\omega$ and Spalart-Allmaras models produce good agreement with respect to the LES data, in both the laminar, transitional, and turbulent regions. A too pronounced minimum $c_{f}$ peak is observed for the Spalart-Allmaras model, which is also present in the other flows considered in the present study (Figs. 2, 3 and 4).

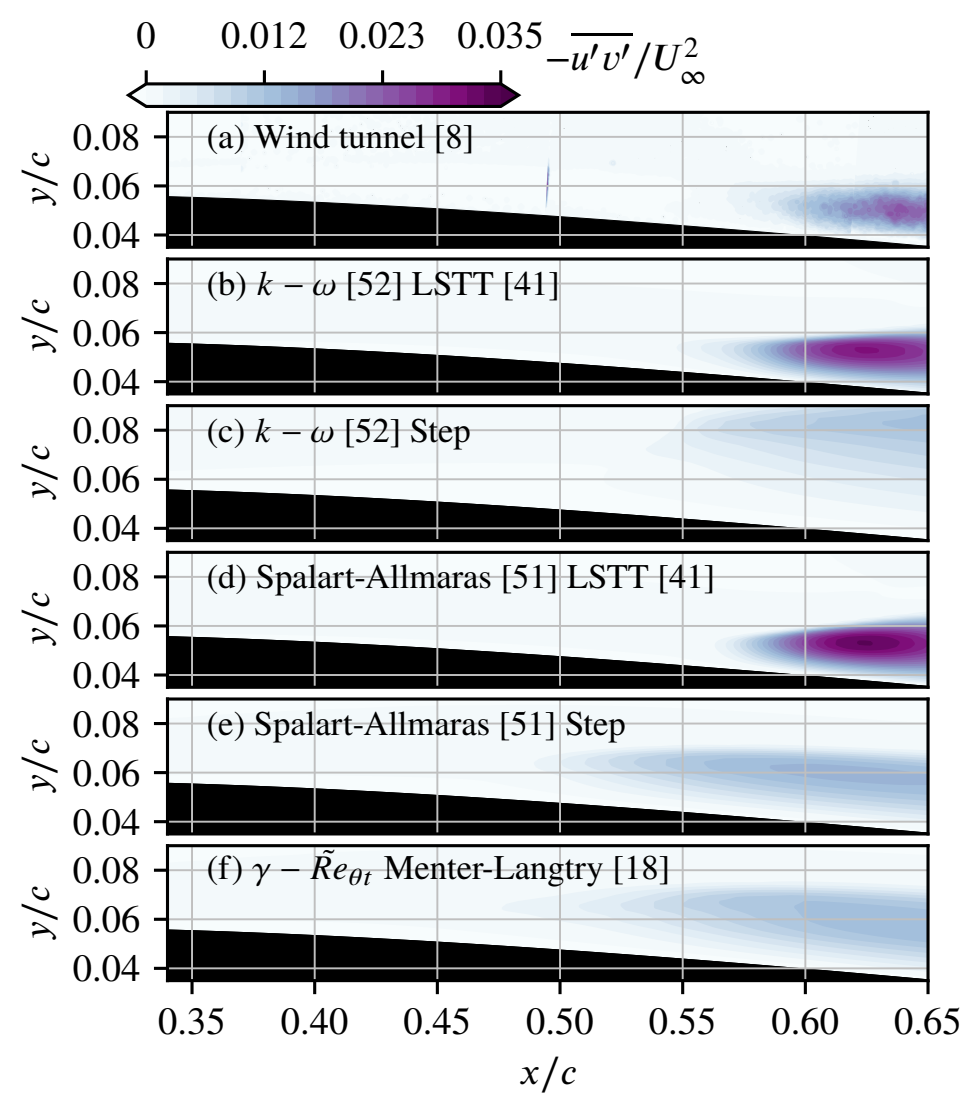

Fig. 7 Comparison of measured (Hain et al. [8]) levels of Reynolds shear-stress $\left(\alpha=4^{\circ}, R e_{c}=66,000\right)$ with computations $\left(\alpha=4^{\circ}, \operatorname{Re}_{c}=\mathbf{6 0 , 0 0 0}\right)$ using different transition triggering strategies $(k-\omega[$ [52] + LSTT [41], SpalartAllmaras [51] + LSTT and $k-\omega[$ [52] with Step activation and shear limiter).

The authors believe that the reason why the LSTT approach is in better agreement with LES data than $\gamma-\tilde{R} e_{\theta t}$ and Step activations, is because LSTT is designed to account for the relatively fast rate of production of Reynolds shear stress in the transitional region, whereas the other approaches underestimate this rate of growth. In order to verify this point, a closer look on the Reynolds shear stress is done in Fig. 7. The Reynolds shear stress $-\overline{u^{\prime} v^{\prime}}$ is plotted using different RANS approaches, and they are compared against the available PIV experimental data of Hain et al. [8]. Although the chord-based Reynolds number of the experiment was slightly different than the RANS computations $(66,000$ versus 60,000$)$, we consider this difference sufficiently low for allowing qualitative comparisons focused on the behavior of the models. In Fig. 7.(b) and77(d) we can observe that LSTT approach predicts similar shear stress when using $k-\omega$ and Spalart-Allmaras models. The overall shape and location of the produced shear stress predicted by the LSTT computations are in good agreement with the experiment (Fig. 7.(a)), although the maximum value is overestimated. On the contrary, the Step approach (Fig. 7(c) and (e)) and Menter-Langtry model (Fig. 7(f)) are in less good agreement with the experimental data, as the predicted values of shear stress are underestimated and the location of its maximum value do not match the location observed in the experiment. Therefore, we may conclude that the reason why $\gamma-\tilde{R} e_{\theta t}$ and Step approaches underestimate the skin-friction downstream of the transition onset may be related to a lack of production rate of shear stress in the transitional region, which is the essential modeling issue addressed by the LSTT approach. 
ILES Galbraith and Visbal [64]

$\triangle$ LES Catalano and Tognaccini [60]
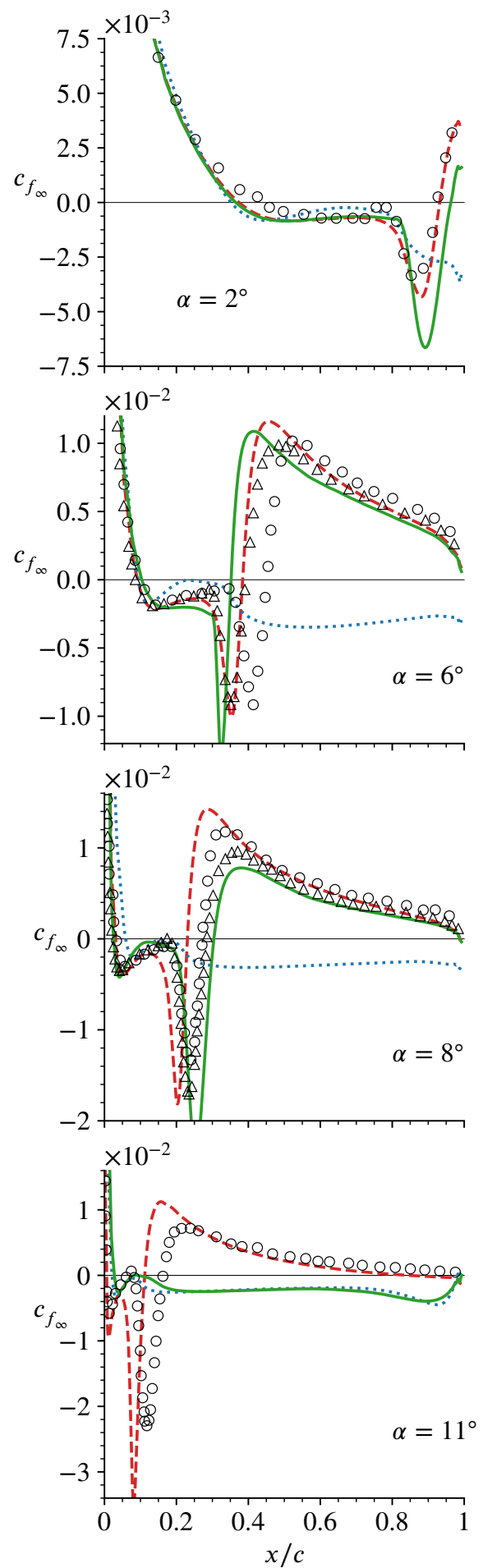

$---k-\omega$ Wilcox (2006) LSTT

$k-\omega$ Wilcox (2006) Step

Spalart-Allmaras LSTT

$C_{p} 0.0$
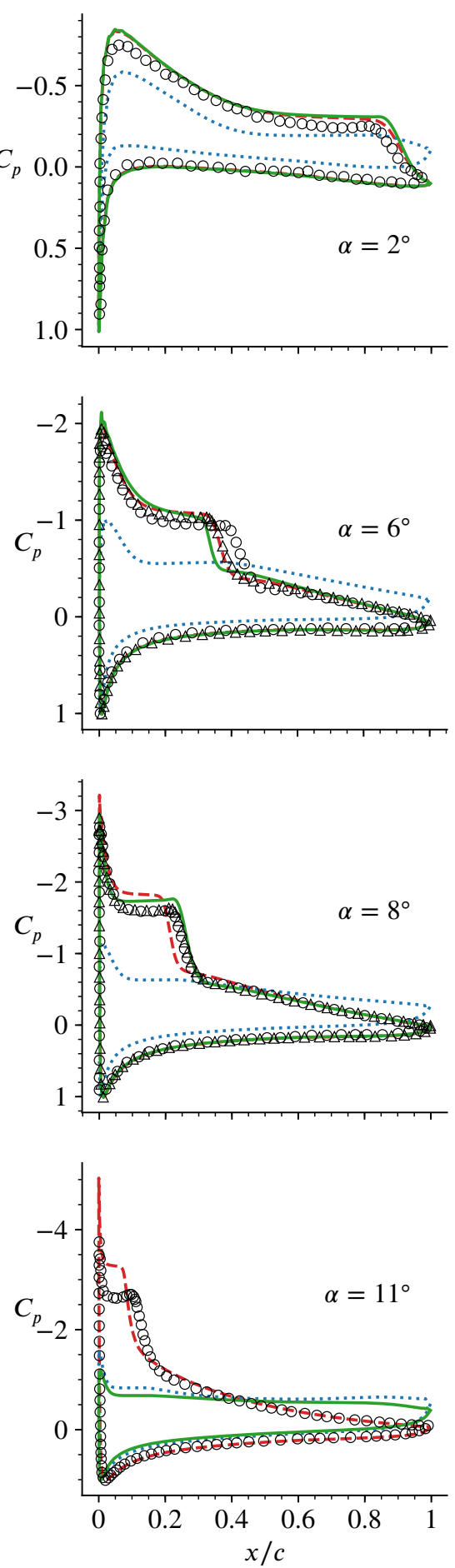

Fig. 8 Comparison of chordwise distributions of skin-friction ( $c_{f_{\infty}}$ on the suction side) and of pressure coefficient $C_{p}$ obtained from different RANS computations with available LES data [60, 64] at different angles of attack $\left(\alpha=2^{\circ}, 6^{\circ}, \mathbf{8}^{\circ}, 1^{\circ}\right)$ at $R e_{c}=60,000$ and $N_{\mathrm{cr}}=11\left(T_{u_{\mathrm{tr}}} \approx 0.03 \%\right)$ 
In Fig. 8 we assess the influence of the angle of attack. With increasing $\alpha$ (Fig. 8), the bubble shrinks from $\sim 55 \% c$ at $\alpha=2^{\circ}$ (separation at $x \approx 0.4 \mathrm{c}$ with reattachment at $x \approx 0.95 \mathrm{c}$ very near the trailing-edge) to $\sim 20 \% \mathrm{c}$ at $\alpha=11^{\circ}$ (separation very near the leading edge with reattachment at $x \approx 0.20 c$ ).

Regarding the importance of the triggering approach, the conclusions are quite similar to those drawn for the baseline case $\left(\alpha=4^{\circ}, R e_{c}=60,000\right)$. Abrupt activation of the turbulence model at the transition onset generally fails, possibly returning stalled flow with an open bubble (Fig. 8) as the prediction using the $k-\omega$ model shows.

On the contrary, the LSTT computations are generally in satisfactory agreement (Fig. 8) with LES data [60, 64]. At $\alpha=2^{\circ}$, the agreement with the LES data of Galbraith and Visbal [64] is excellent for $k-\omega$ and quite good for Spalart-Allmaras. At $\alpha=6^{\circ}$, there is a slight scatter between the 2 sets of LES data [60, 64], the LSTT computations being in close agreement with Catalano and Tognaccini [60]. At $\alpha=8^{\circ}$, where the 2 sets of LES data are in better agreement one with another, the $k-\omega+$ LSTT model suffers from the prediction by the criteria of too early a transition-onset location, which induces an earlier negative $c_{f_{\infty}}$ peak and earlier reattachment, also resulting in a slight discrepancy of the pressure plateau associated with the LSB. The same remarks apply to the $\alpha=11^{\circ}$ case (Fig. 8) regarding $k-\omega$ LSTT. On the contrary, at $\alpha=8^{\circ}$ the Spalart-Allmaras LSTT model does not produce a too upstream transition onset prediction. However, let us highlight that at $\alpha=11^{\circ}$ the Spalart-Allmaras LSTT model predicts a stalled condition. Hence, we believe that the reason why at $\alpha=8^{\circ}$ the $k-\omega$ LSTT predicts a too early transition and Spalart-Allmaras LSTT does not, is because Spalart-Allmaras at $\alpha=8^{\circ}$ is very close to bubble bursting.

Despite the fact that Spalart-Allmaras LSTT model does not successfully predict the LES data at this near-stall condition $\left(\alpha=11^{\circ}\right)$, this prediction shows that LSTT activation (which boosts Reynolds stress production) does not inhibit the capacity of the model to predict stall and bubble bursting. Contrarily, by successfully predicting moderate-to-high angles of attack LSBs, the LSTT approach may contribute to enhancing the RANS ability to capture the airfoil's stall threshold.
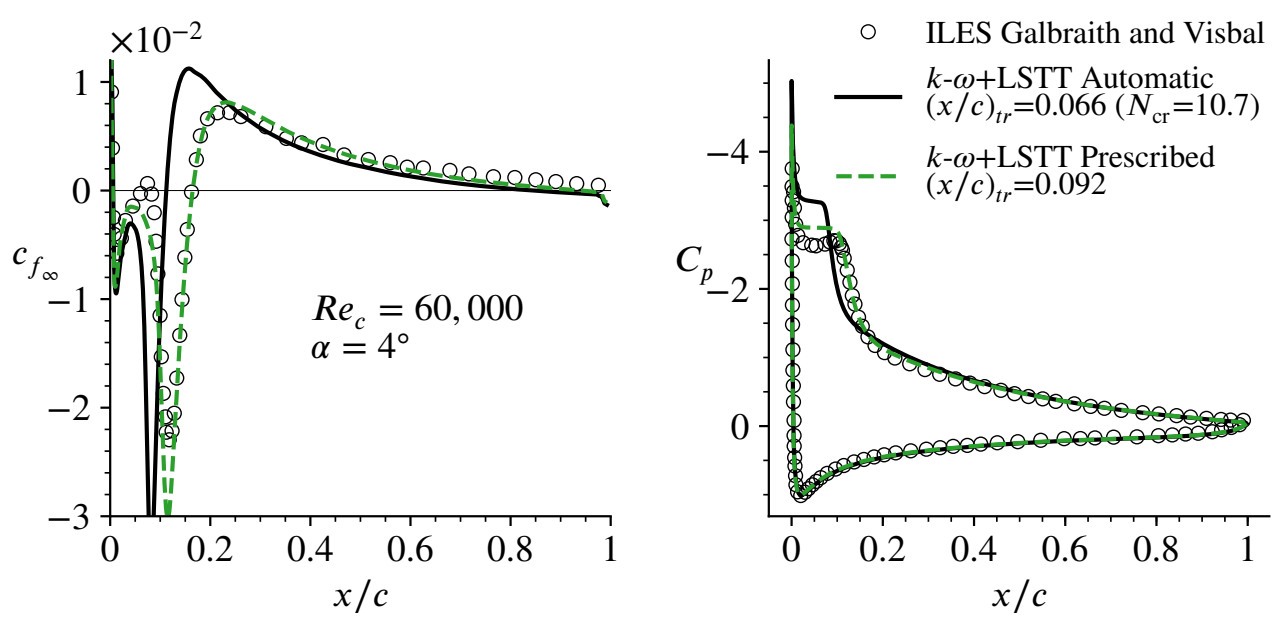

Fig. 9 Comparison of $c_{f_{\infty}}$ (on the suction side) and $C_{p}$ obtained from LES [64] against RANS computations $(k-\omega[$ [52] + LSTT [41]) using automatic or prescribed transition onset location.

In order to determine the reasons for the observed discrepancies between the $k-\omega+$ LSTT predictions and the LES data [60, 64] at the higher $\alpha \in\left\{8^{\circ}, 11^{\circ}\right\}$ (Fig. 8) we performed an additional calculation at $\alpha=11^{\circ}$ (Fig. 9), where the transition onset was prescribed at $x_{t r}=0.092 \mathrm{c}$ further downstream of the criteria-predicted $\left(N_{c r} \approx 11\right)$ location of transition onset at $x_{t r}=0.066 c$. This $a d$ hoc adjustment of the transition onset location greatly improves the agreement with LES: the location of the negative $c_{f_{\infty}}$ peak and of reattachment are in excellent agreement with the LES computations, and these results in a substantially improved prediction of the pressure plateau in the $C_{p}$ distributions (Fig. 9).

These results demonstrate that the LSTT approach is quite successful in controlling the turbulence built-up in the transitional region, provided that the correct $x_{\text {tr }}$ is obtained by the transition criteria. Inversely, the increased discrepancy of the $k-\omega+$ LSTT computations with LES as $\alpha$ increases is principally associated with the inaccurate (early) prediction of transition onset. This would imply that with increasing $\alpha, N_{c r}$ should also increase. Mack's correlation would then suggest that the effective [65, 66] $T_{u_{\mathrm{tr}}}$ decreases. An argument in support of this supposition is that as the laminar separation point moves upstream, closer to the stagnation point, with increasing $\alpha$, the boundary-layer thickness at 
separation $\delta_{\text {sep }}$ is modified, and so does the receptivity of the boundary-layer to a given spectrum of external turbulence, in line with theoretical arguments [65, 66] corroborated by numerical stability calculations [67]. This is naturally incorporated in LES dynamics but not in Mack's correlation (Eq. 7) which assumes a monotonic bijection $N_{c r}\left(T_{u_{\mathrm{tr}}}\right)$. With regard to the previous results (Figs. 8, 9), it may be surmised that, as $\alpha$ increases and the LSB size shrinks, increased diffusion of momentum by the Reynolds shear-stress $-\overline{u^{\prime} v^{\prime}}$ is necessary to compensate the high inertial forces of the flow and the increased deceleration associated with the adverse local pressure gradient. It is precisely because the LSTT approach, by boosting production of turbulence and inhibiting the shear limiter of $k-\omega$ models until after fully turbulent conditions are reached, achieves a better prediction of the Reynolds shear-stress $-\overline{u^{\prime} v^{\prime}}$ (Fig. 7 ) that it performs better than the Step-activated approaches, significantly improving the predictive accuracy of the bubble's topology. The convection effect of the correct prediction of the transitional region in the LSTT approach translates into proper estimation of the $c_{f}$ values downstream of the bubble.

\section{T106C turbine blade}

The T106C test case is based on the experiments performed by Michalek et al. [68]. They studied the low-pressure high-lift turbine blade T106C on a linear cascade in the VKI S1/C high-speed wind tunnel operating at exit Mach number of $M=0.65$. Multiple cases were analyzed, particularly for varying chord-based Reynolds number and free-stream turbulence levels. In this study, two configurations are considered as shown in Fig. 10, the $R e_{c}=120,000$ and $R e_{c}=250,000$ cases, both at $T_{u_{\infty}}=0.9 \%$. The $k-\omega$ [52] LSTT prediction is compared against the $\gamma-\tilde{R} e_{\theta t}$ model with Content-Houdeville [69] correlations with $T_{u}=0.9 \% s$. For the LSTT approach, the AHD-Gleyzes [44, 53] criteria were employed, initially using $N_{c r}=2.9\left(T_{u}=0.9 \%\right)$ and then adjusted to $N_{c r}=10$ in order to fit the experimental data transition onset for assessing the turbulence model's activation.
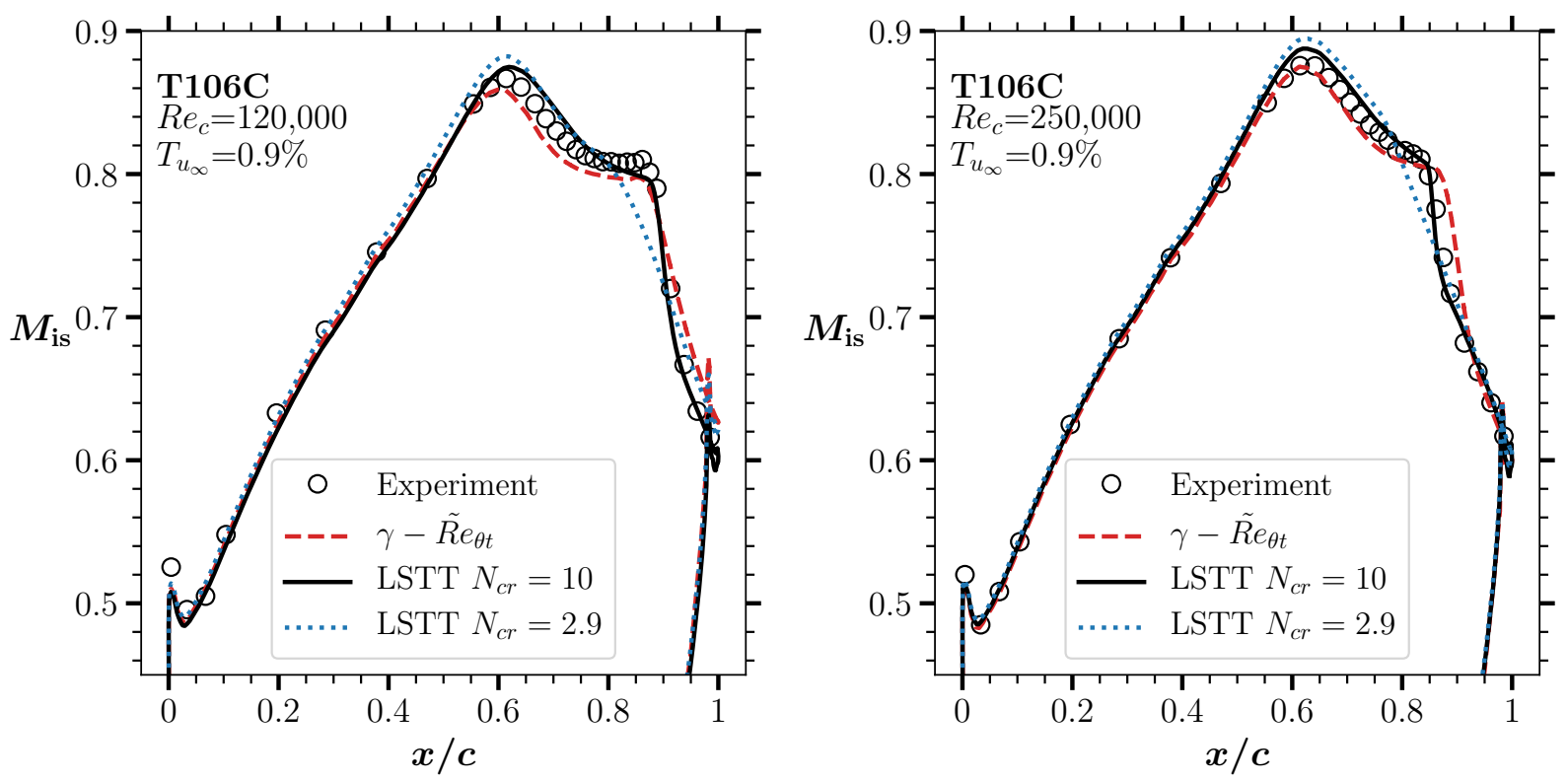

Fig. 10 Comparison of isentropic Mach, $M_{\mathrm{is}}$, over the suction side of the T106C turbine blade, using $k-\omega$ [52] LSTT model and Menter-Langrty $\gamma-\tilde{R e} e_{\theta t}$ (with $T_{u}=0.9 \%$ ) [17, [0] model with Content-Houdeville [69] correlations, against available wind tunnel experimental data.

In Fig. 10 we present the chordwise distribution of isentropic Mach $M_{\text {is }}$ of the suction side of the blade. The $\gamma-\tilde{R} e_{\theta t}$ model produces good predictions of this flow. We observe only a slightly slow recovery from the end of the plateau of $M_{\mathrm{is}}$, which may translate into an overestimation of the bubble size. On the other hand, the LSTT automatic transition prediction with the prescribed turbulence value of $T_{u}=0.9 \%\left(N_{c r}=2.9\right)$ predicts a transition onset located at $x_{\text {tr }} / c \approx 0.80$, which is quite far upstream of the observed experimental transition onset $\left(x_{\mathrm{tr}} / c \approx 0.85\right)$. As a result, LSTT computations using $N_{c r}=2.9$ fail to predict the LSB. In order to assess the influence on the value of $N_{c r}$, a sweep of this parameter was done. It was found that using $N_{c r}=10$ the LSTT computations yield very good predictions with respect to experimental data. However, $N_{c r}=10$ is associated with very low turbulence level $\left(T_{u} \approx 0.046\right)$ following Mack's 
relationship. This last aspect is unsatisfactory, as accurate transition criteria should provide correct transition onset prediction for measured experimental turbulence level. This inaccuracy may be caused by one or several of the following points: (a) employed transition criteria may not be precise enough for this flow condition, (b) Mack's relationship may not relate precisely enough the freestream turbulence level to the actual turbulence breakdown threshold and (c) the measured value $T_{u}=0.9 \%$ may be much higher than the actual turbulence level value at the laminar boundary-layer edge, $T_{u_{\delta}}$, where the receptivity mechanism takes place.

Considering the LSTT computation at $N_{c r}=10$, we can evaluate the turbulence model's activation precision with respect to $\gamma-\tilde{R} e_{\theta t}$ and the experimental data. Hence, we can observe that LSTT approach yields a steeper recovery after the plateau than $\gamma-\tilde{R} e_{\theta t}$ prediction. This results in better agreement with experimental data. A similar effect was observed and discussed upon $C_{p}$ distribution of the SD7003 airfoil in Fig. 6 Thus, in a similar fashion as concluded in the analysis of Fig. 6, the fact that LSTT predicts a steeper and more accurate recovery after the plateau (Fig. 10) may be due to LSTT better accounting for the smooth and important production of Reynolds shear stress in the transitional region.

\section{Conclusion}

Several transitional RANS approaches applied to a collection of low Reynolds number aerodynamic flows were assessed. The recently proposed non-local Laminar Separation Transition Triggering (LSTT) [41] approach, originally designed for use with $k-\omega[35,52,71,72]$ turbulence models and transition criteria [44, 53], was extended to the one-equation Spalart-Allmaras [51] turbulence model. The LSTT approach was compared to typical Step off-on [34, 39] activation of RANS turbulence models and transport equation-based $\gamma-\tilde{R e} e_{\theta t}[17,18,69,70,73]$ transitional model. RANS computations were systematically compared against available published DNS [13, 47], LES [60], ILES [59, 64] or experimental [8,68] data.

Results show that LSTT approach produces an enhancement of the precision of the RANS predictions, particularly for low Reynolds number flows. The present analysis suggests that Step activations and $\gamma-\tilde{R e} e_{\theta t}$ model tend to underestimate the rate of growth of turbulent shear stress in the transitional region, which provokes a downstream delay of the reattachment point or may even produce an unphysical stalled condition. This scenario is problematic at high angle-of-attack and low Reynolds number flows, where the accurate prediction of the laminar separation bubble determines the RANS model's ability to predict the bubble bursting threshold, which is commonly the main cause of stall. It is inferred that proper modeling of the LSB is also of fundamental interest for accurately predicting the downstream turbulent boundary-layer profile, notwithstanding the natural limits of the original turbulence model's behavior [58].

As the LSTT approach requires the use of non-local transition criteria, this means that the accuracy and robustness of the method depends on those of the transition criteria. This may yield several advantages, like the use of accurate physics-based correlations using integral boundary-layer parameters especially suited for capturing each transition mechanism (natural, separation-induced, crossflow, bypass...). But it may also yield several disadvantages, like a more important implementation effort than local transport equation-based approaches [73] particularly for massively parallel unstructured grids. The implementation of non-local transition criteria has proven to be suitable for massively parallel structured codes [45], but its extension to unstructured grids is still under work.

This work shows that one of the main assets of the LSTT approach is the use of the non-local length scale $s_{\text {tr }}-s_{\text {sep }}$. This scale, which was also used by Lian and Shyy [36], seems to be an appropriate choice in order to properly control the non-equilibrium region where the boost of turbulence is promoted. The LSTT approach shows that this scale can be used for appropriately delaying shear limiters that may produce an unfavorable effect in the transitional region. Basing the turbulence activation mechanisms upon this lengthscale allows for proper dependence upon physical conditions like $T_{u}$, $\alpha$ and $R e_{c}$. The assumption performed by LSTT which consists in replacing the commonly accepted intermittency factor, $\gamma$, as the main mechanism responsible for the turbulence model's activation, by the empirical weighting coefficient $f^{\text {tr }}$, allowed to overcome irrelevant physical constraints inherent to $\gamma$. Such constraints are its physical bounds $(\gamma \in[0,1])$, its presumed universality as a physical quantity (particularly problematic when used for triggering different turbulence models) or even the controversy on how $\gamma$ (issued from conditional averaging [74]) interacts with the unconditioned quantities of a RANS framework. On the contrary, this study shows that empirical $f^{\mathrm{tr}}(\neq \gamma)$ functions designed and calibrated $a d-h o c$ for each considered turbulence model significantly improves the RANS predictions.

A number of perspectives can be inferred from the present work. First, the LSTT approach may be extended to other turbulence models and this may contribute to further understanding the necessity of boosting the turbulent shear stress in the transitional region. Second, modeling work can be performed in order to transform LSTT into a 
purely local formulation, ready to use with general unstructured grids. Third, a thorough evaluation of the prediction capabilities of the bubble bursting phenomenon, even for dynamic motion of the airfoil, would be desirable as this phenomenon is related to stall. And last, further effort on improving the accuracy and robustness of transition criteria is of great importance in order to produce accurate RANS predictions, as was shown in this study. A reassessment on the Mack's relationship may be desirable in order to better account for the critical total amplification value determining the turbulence breakdown threshold for varying inflow turbulence spectra, including those existing in DNS and ILES.

\section{Acknowledgments}

The authors would like to acknowledge the great support and useful insights of Prof. G. Gerolymos from Sorbonne Universités and to our colleagues at the DAAA and DMPE Departments at ONERA. Special thanks to Mr. Hain, Mr. Kähler and Mr. Radespiel for sharing their PIV data with us. The simulations run in this study were executed using the software elsA [75], which is issued of the three-party agreement between AIRBUS, SAFRAN, and ONERA. All the preand post-process tasks were performed using the Cassiopee [76] software, and all the figures presented in this work were produced with the Matplotlib [77] library. This work was completely funded by ONERA in the framework of the $\mathrm{PhD}$ thesis of the first author.

\section{A. Appendix: Stabilization of Gleyzes Transition Criterion}

As the Gleyzes's [44] correlation shows (5), the criterion implicitly assumes a monotonically increasing $R e_{\theta}$ with respect to the curvilinear abscissa $s$. However, in general this is not verified, particularly for deeply detached boundary-layers $(H \gtrsim 5)$, as the evolution of $R e_{\theta}$ with respect to $s$ may stagnate, or even decrease [11]. Under these circumstances, $N(s)$ value issued from the integration of (5) may stagnate, or even decrease, systematically delaying downstream the transition onset location and compromising the RANS simulation. This is not a physically desired behavior. On the contrary, we would rather expect the inverse behavior, viz. a slight upstream displacement of the transition onset, as under deeply detached conditions the flow is very instable, promoting a more rapid growth of perturbations leading to turbulence.

In order to solve this issue, the authors suggest the use of an algebraic extrapolation technique designed to guarantee the monotonicity of the integrand of (5). This also enhances the numerical convergence of the simulation, as the transition onset prediction becomes less sensitive to the boundary-layer quantities at the transition onset location. The authors suggest that this sensitivity, which is particularly pronounced in recirculating flow, is one of the most common causes of transition onset location oscillations leading to poor convergence.

In the following, the aforementioned algebraic extrapolation technique is presented. The idea consists in replacing the co-processed $H(s)$ and $\operatorname{Re}_{\theta}(s)$ values by a mathematical approximation $\phi$ and $\psi$ constructed from the exact boundary-layer values at several points $s^{P_{i}}$ located in the laminar region, which we denote control points,

$$
\begin{gathered}
H(s) \approx \phi\left(s, H\left(s^{P_{i}}\right)\right) \\
\operatorname{Re}_{\theta}(s) \approx \psi\left(s, \operatorname{Re}_{\theta}\left(s^{P_{i}}\right)\right)
\end{gathered}
$$

The approximation functions $\phi$ and $\psi$ shall be monotonically increasing. They shall extrapolate precisely enough the $H$ and $\operatorname{Re}_{\theta}$ laminar trend from the separation point. The functions shall be reasonably independent on the choice of the control points $s^{P_{i}}$.

We suggest exactly fitting $\phi$ to a concave parabola using three characteristic values, $H^{P_{1}}=3, H^{P_{2}}=4$ and $H^{P_{3}}=5$, used to infer the control points locations by assuming local bijection,

$$
\begin{aligned}
& s^{P_{1}}=s\left(H=H^{P_{1}}\right) \\
& s^{P_{2}}=s\left(H=H^{P_{2}}\right) \\
& s^{P_{3}}=s\left(H=H^{P_{3}}\right)
\end{aligned}
$$

We suggest fitting $\psi$ to a monotonically increasing power function,

$$
\psi=c_{1} s^{\lambda}+c_{2}
$$


Where the constants $c_{1}$ and $c_{2}$ are found by introducing the set $\left\{\left(s^{P_{1}}, \operatorname{Re}_{\theta}\left(s^{P_{1}}\right)\right),\left(s^{P_{2}}, \operatorname{Re}_{\theta}\left(s^{P_{2}}\right)\right)\right\}$ into $[13]$, and solving for $c_{1}$ and $c_{2}$. We found that extrapolations of the Gleyzes integral function (6) is quite insensitive to the value of $\lambda$, provided it yields a low enough value. We suggest using $\lambda=10^{-2}$.

For concluding the presentation of this extrapolation strategy, we must tell the algorithm how to behave in case one or several requirements cannot be verified or if one or several control points cannot be found. In the event one of the three $s^{P_{i}}$ points cannot be computed (due to, e.g. a mildly detached boundary layer where $H_{\max }<5$ ) an attempt can be done to produce a monotonically increasing linear fit with the two remaining control points. In case that a linear fit is not possible or if the resulting parabola fit for $\phi$ is convex, the algorithm should use the computed boundary-layer $H$ and $R e_{\theta}$ values in (6). Following this rationale, the stabilization technique presented in this appendix shall only intervene in highly detached boundary-layers, which actually constitute the problematic scenario where the standard Gleyzes [44] formulation (5) fails.

\section{References}

[1] Tani, I., "Low-speed flows involving bubble separations," Progress in Aerospace Sciences, Vol. 5, 1964, pp. 70 - 103. doi:10.1016/0376-0421(64)90004-1.

[2] Richez, F., Mary, I., Gleize, V., and Basdevant, C., "Near stall simulation of the flow around an airfoil using zonal RANS/LES coupling method," Computers \& Fluids, Vol. 37, No. 7, 2008, pp. 857 - 866. doi:10.1016/j.compfluid.2007.03.016.

[3] Raffel, M., Favier, D., Berton, E., Rondot, C., Nsimba, M., and Geissler, W., "Micro-PIV and ELDV wind tunnel investigations of the laminar separation bubble above a helicopter blade tip," 6th International Symposium on Particle Image Velocimetry, Vol. 17, IOP Publishing Ltd, Pasadena, California, USA, 2005, pp. 1-13. doi:10.1088/0957-0233/17/7/003.

[4] Pines, D. J., and Bohorquez, F., "Challenges Facing Future Micro-Air-Vehicle Development," Journal of Aircraft, Vol. 43, No. 2, 2006, pp. 290-305. doi:10.2514/1.4922.

[5] Somers, D. M., "Design and Experimental Results for the S809 Airfoil," Tech. Rep. NREL/SR-440-6918, National Renewable Energy Laboratory, Golden, Colorado, USA, January 1997.

[6] Gaster, M., "The Structure and Behaviour of Laminar Separation Bubbles," Tech. Rep. R\&M No. 3595, Aeronautical Research Council, 1969.

[7] Malkiel, E., and Mayle, R. E., “Transition in a Separation Bubble,” Journal of Turbomachinery, Vol. 118, No. 4, 1996, pp. 1-11. doi:10.1115/1.2840931.

[8] Hain, R., Kähler, C. J., and Radespiel, R., "Dynamics of laminar separation bubbles at low-Reynolds-number aerofoils," Journal of Fluid Mechanics, Vol. 630, 2009, pp. 129-153. doi:10.1017/S0022112009006661.

[9] Crabtree, L., “The Formation of Regions of Separated Flow on Wing Surfaces,” Tech. Rep. 3122, ARC R\&M, London, 1959.

[10] Sanz, W., and Platzer, M. F., “On the Navier-Stokes Calculation of Separation Bubbles With a New Transition Model,” Journal of Turbomachinery, Vol. 120, No. 1, 1998, pp. 36-42. doi:10.1115/1.2841385.

[11] Horton, H. P., "Laminar separation bubbles in two- and three-dimensional incompressible flow," Ph.D. thesis, University of London, 1968.

[12] Carmichael, B., "Low Reynolds number airfoil survey,” Tech. Rep. NASA-CR-165803, NASA, 1982.

[13] Jones, L. E., Sandberg, R. D., and Sandham, N. D., "Direct numerical simulations of forced and unforced separation bubbles on an airfoil at incidence," Journal of Fluid Mechanics, Vol. 602, 2008, pp. 175-207. doi:10.1017/S0022112008000864.

[14] Yarusevych, S., and Kotsonis, M., "Steady and transient response of a laminar separation bubble to controlled disturbances," Journal of Fluid Mechanics, Vol. 813, 2017, pp. 955-990. doi:10.1017/jfm.2016.848.

[15] Michelis, T., Yarusevych, S., and Kotsonis, M., "On the origin of spanwise vortex deformations in laminar separation bubbles," Journal of Fluid Mechanics, Vol. 841, 2018, pp. 81-108. doi:10.1017/jfm.2018.91.

[16] Dick, E., and Kubacki, S., “Transition models for turbomachinery boundary layer flows : a review," International Journal Of Turbomachinery Propulsion And Power, Vol. 2, No. 2, 2017, p. 44. doi:10.3390/ijtpp2020004. 
[17] Langtry, R. B., Menter, F. R., Likki, S. R., Suzen, Y. B., Huang, P. G., and Völker, S., "A Correlation-Based Transition Model Using Local Variables - Part II: Test Cases and Industrial Applications," Journal of Turbomachinery, Vol. 128, 2006, pp. 423-434. doi:10.1115/1.2184353.

[18] Langtry, R. B., and Menter, F. R., "Correlation-Based Transition Modeling for Unstructured Parallelized Computational Fluid Dynamics Codes,” AIAA Journal, Vol. 47, No. 2, 2009, pp. 2894-2906. doi:10.2514/1.42362.

[19] Abu-Ghannam, B. J., and Shaw, R., "Natural transition of boundary-layers - The effects of turbulence, pressure gradient, and flow history," IMechE J. Mech. Eng. Sci., Vol. 22, No. 5, 1980, pp. 213-228. doi:10.1243/JMES_JOUR_1980_022_043_02.

[20] Dhawan, S., and Narasimha, R., "Some properties of boundary layer flow during the transition from laminar to turbulent motion,” Journal of Fluid Mechanics, Vol. 3, No. 4, 1958, pp. 418-436. doi:10.1017/S0022112058000094.

[21] Durbin, P., “An intermittency model for bypass transition,” International Journal of Heat and Fluid Flow, Vol. 36, 2012 , pp. 1-6. doi:10.1016/j.ijheatfluidflow.2012.03.001.

[22] Menter, F. R., Smirnov, P. E., Liu, T., and Avancha, R., “A One-Equation Local Correlation-Based Transition Model,” Flow, Turbulence and Combustion, Vol. 95, No. 4, 2015, pp. 583-619. doi:10.1007/s10494-015-9622-4.

[23] Walters, D. K., and Leylek, J. H., “A New Model for Boundary Layer Transition Using a Single-Point RANS Approach,” Journal of Turbomachinery, Vol. 126, No. 1, 2004, pp. 193-202. doi:10.1115/1.1622709.

[24] Walters, D. K., and Leylek, J. H., "Computational Fluid Dynamics Study of Wake-Induced Transition on a Compressor-Like Flat Plate," Journal of Turbomachinery, Vol. 127, No. 1, 2005, pp. 52-63. doi:10.1115/1.1791650.

[25] Walters, and Cokljat, "A Three-Equation Eddy-Viscosity Model for Reynolds-Averaged Navier-Stokes Simulations of Transitional Flow," Journal of Fluids Engineering, Vol. 130, No. 12, 2008. doi:10.1115/1.2979230.

[26] Mayle, R. E., and Schulz, A., “The path to predicting bypass transition,” ASME J. Turbom., Vol. 119, 1997 , pp. 405-411. doi:10.1115/1.2841139.

[27] Tani, I., "Boundary-later transition,” Ann. Rev. Fluid Mech., Vol. 1, 1969, pp. 169-196.

[28] Gerolymos, G. A., and Vallet, I., “The dissipation tensor $\varepsilon_{i j}$ in wall turbulence,” J. Fluid Mech., Vol. 807, 2016 , pp. $386-418$. doi:10.1017/jfm.2016.610.

[29] Kubacki, S., and Dick, E., "An algebraic intermittency model for bypass, separation-induced and wake-induced transition," International Journal of Heat and Fluid Flow, Vol. 62, 2016, pp. 344-361. doi:10.1016/j.ijheatfluidflow.2016.09.013.

[30] Minot, A., Salah El-Din, I., Barrier, R., Boniface, J. C., and Marty, I., "Improvement of Laminar-Turbulent Transition Modeling Within a Low-Pressure Turbine," ASME Turbo Expo 2016: Turbomachinery Technical Conference and Exposition, Vol. 2C, ASME, Seoul, South Korea, 2016. doi:10.1115/GT2016-57637.

[31] Colonia, S., Leble, V., Steijl, R., and Barakos, G., "Assessment and Calibration of the $\gamma$-Equation Transtion Model at Low Mach,” AIAA Journal, Vol. 55, No. 4, 2017, pp. 1126-1139. doi:10.2514/1.J055403.

[32] de Rosa, D., and Catalano, P., "Validation of Intermittency Model for Transition Prediction in a RANS Flow Solver," AIAA Aerospace Sciences Meeting, AIAA, 2018. doi:10.2514/6.2018-1043.

[33] Yuan, W., Khalid, M., Windte, J., Scholz, U., and Radespiel, R., “An Investigation of Low-Reynolds-Number Flows past Airfoils,” 23rd AIAA Applied Aerodynamics Conference, Toronto, 2005. doi:10.2514/6.2005-4607.

[34] Windte, J., Scholz, U., and Radespiel, R., "Validation of the RANS-simulation of laminar separation bubbles on airfoils," Aerospace Science and Technology, Vol. 10, No. 6, 2006, pp. 484-494. doi:10.1016/j.ast.2006.03.008.

[35] Menter, F. R., “Two-equation eddy-viscosity turbulence models for engineering applications," AIAA Journal, Vol. 32, No. 8, 1994, pp. 1598-1605. doi:10.2514/3.12149.

[36] Lian, Y., and Shyy, W., "Laminar-Turbulent Transition of a Low Reynolds Number Rigid or Flexible Airfoil," AIAA Journal, Vol. 45, No. 7, 2007, pp. 1501-1513. doi:10.2514/1.25812.

[37] Wilcox, D. C., "Simulation of Transition with a Two-Equation Turbulence Model," AIAA Journal, Vol. 32, No. 2, 1994, pp. 247-255. doi:10.2514/3.59994. 
[38] Smith, A., and Gamberoni, N., "Transition, Pressure Gradient and Stability Theory," Tech. Rep. ES 26388, Douglas Aircraft Company, El Segundo, California, USA, Aug. 1956.

[39] Catalano, P., and Tognaccini, R., “Turbulence Modeling for Low-Reynolds-Number Flows,” AIAA Journal, Vol. 48, No. 8, 2010, pp. 1673-1685. doi:10.2514/1.J050067.

[40] Catalano, P., Mele, B., and Tognaccini, R., "A Numerical Method to Detect Laminar Separation Bubbles over Airfoils," $31 s t$ AIAA Applied Aerodynamics Conference, AIAA, San Diego, CA, USA, 2013. doi:10.2514/6.2013-2528.

[41] Bernardos, L., Richez, F., Gleize, V., and Gerolymos, G. A., "Algebraic Nonlocal Transition Modeling of Laminar Separation Bubbles using k- $\omega$ Turbulence Models,” AIAA Journal, Vol. 57, No. 2, 2019, pp. 553-565. doi:10.2514/1.J057734.

[42] Roberts, W. B., "Calculation of Laminar Separation Bubbles and Their Effect on Airfoil Performance," AAIA Journal, Vol. 18, No. 1, 1980, pp. 25-31.

[43] Arnal, D., Habiballah, M., and Coustols, E., "Laminar Instability Theory And Transition Criteria In Two and Three-Dimensional Flow," Recherche Aérospatiale (ISSN 0379-380X), Vol. 2, 1984, pp. 125-143.

[44] Gleyzes, C., Cousteix, J., and Bonnet, J. L., "A Calculation Method of Leading-Edge Separation Bubbles,” Numerical and Physical Aspects of Aerodynamic Flows II, edited by T. Cebeci, Springer Berlin Heidelberg, Berlin, Heidelberg, 1984, pp. 173-192. doi:10.1007/978-3-662-09014-5_10.

[45] Cliquet, J., Houdeville, R., and Arnal, D., "Application of Laminar-Turbulent Transition Criteria in Navier-Stokes Computations," AIAA Journal, Vol. 46, No. 5, 2008, pp. 1182-1190. doi:10.2514/1.30215.

[46] Laurent, C., Mary, I., Gleize, V., Lerat, A., and Arnal, D., "RANS Modeling of a Transitional Laminar Separation Bubble on a Flat Plate with DNS Database," AIAA, Chicago, Illinois, 2010. doi:10.2514/6.2010-4444.

[47] Laurent, C., Mary, I., Gleize, V., Lerat, A., and Arnal, D., "DNS database of a transitional separation bubble on a flat plate and application to RANS modeling validation,” Computers \& Fluids, Vol. 61, 2012, pp. 21 - 30. doi:10.1016/j.compfluid.2011.07.011, onera Scientific Day.

[48] Arnal, D., "Laminar-turbulent transition problems in supersonic and hypersonic flow," Tech. Rep. 761, AGARD, 1988.

[49] Wilcox, D. C., Turbulence Modeling for CFD, $3^{\text {rd }}$ ed., DCW Industries, La Cañada, California, 2006.

[50] Wilcox, D. C., "A Half Century Historical Review of the k- $\omega$ Model," 29th Aerospace Sciences Meeting, edited by AIAA, Reno, Nevada, 1991. doi:10.2514/6.1991-615.

[51] Spalart, and Allmaras, "A one-equation turbulence model for aerodynamic flows," American Institute of Aeronautics and Astronautics, 1992. doi:doi:10.2514/6.1992-439.

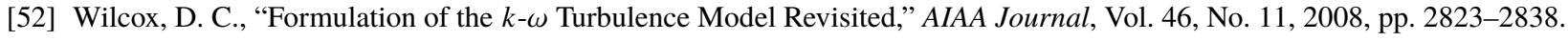
doi:10.2514/1.36541.

[53] Arnal, D., "Description and Prediction of Transition in Two-Dimensional Incompressible Flow," Special Course on Stability and Transition of Laminar Flow, 709, NATO-AGARD, 1984, Chap. 2.

[54] Arnal, D., "Boundary Layer Transition: Predictions Based on Linear Theory," Special Course on Progress in Transition Modelling, 793, AGARD, 1994, Chap. 2, pp. 1-63.

[55] Mack, L. M., “Transition prediction and linear stability theory,” Tech. Rep. CP 224, 1.1-1.22, AGARD, 1977.

[56] Perraud, J., Deniau, H., and Casalis, G., "Overview Of Transition Prediction Tools In The elsA Software," 6th European Conference on Computational Fluid Dynamics, IACM and ECCOMAS, HAL, Barcelona, Spain, 2014.

[57] Herbst, S., Kähler, C., and Hain, R., "Influence of large-scale free-stream turbulence on an SD7003 airfoil at low Reynolds numbers," AIAA AVIATION Forum, Atlanta, USA, 2018. doi:10.2514/6.2018-3490.

[58] Bernardos, L., Richez, F., Gleize, V., and Gerolymos, G. A., "On the $k-\omega$ models behavior in the boundary-layer downstream of a short transitional separation bubble," AIAA Aerospace Sciences Meeting, AIAA, Kissimmee, Florida, USA, 2018. doi:10.2514/6.2018-0376.

[59] Alferez, N., Mary, I., and Lamballais, E., "Study of Stall Development Around an Airfoil by Means of High Fidelity Large Eddy Simulation," Flow, Turbulence and Combustion, Vol. 91, No. 3, 2013, pp. 623-641. doi:10.1007/s10494-013-9483-7. 
[60] Catalano, P., and Tognaccini, R., "Influence of Free-stream Turbulence on Simulations of Laminar Separation Bubbles," 47th AIAA Aerospace Sciences Meeting Including The New Horizons Forum and Aerospace Exposition, AIAA, Orlando, Florida, USA, 2009. doi:10.2514/6.2009-1471.

[61] Visbal, M. R., "High-Fidelity Simulation of Transitional Flows past a Plunging Airfoil," AIAA Journal, Vol. 47, No. 11, 2009, pp. 2685-2697. doi:10.2514/1.43038.

[62] Breuer, M., "Effect of Inflow Turbulence on an Airfoil Flow with Laminar Separation Bubble: An LES Study," Flow, Turbulence and Combustion, 2018. doi:10.1007/s10494-017-9890-2.

[63] Selig, M. S., Guglielmo, J. J., Broeren, A. P., and Giguère, P., Summary of Low-Speed Airfoil Data, Vol. 1, SoarTech Publications, Virginia Beach, VA, 1995.

[64] Galbraith, M. C., and Visbal, M. R., "Implicit Large Eddy Simulation of Low-Reynolds-Number Transitional Flow Past the SD7003 Airfoil," 40th Fluid Dynamics Conference and Exhibit, AIAA, Chicago, Illinois, USA, 2010. doi:10.2514/6.2010-4737.

[65] Dullenkopf, K., and Mayle, R., "An account of free-stream-turbulence lengthscale on laminar heat transfer," ASME Journal of Turbomachinery, Vol. 117, 1995, pp. 401-406. doi:10.1115/94-GT-174.

[66] Mayle, R. E., Dullenkopf, K., and Schulz, A., "The Turbulence That Matters," ASME Journal of Turbomachinery, Vol. 120, No. 3, 1998, pp. 402-409. doi:10.1115/97-GT-274.

[67] Vermeersch, O., and Arnal, D., "Klebanoff-Mode Modeling and Bypass-Transition Prediction," AIAA Journal, Vol. 48, No. 11, 2010, pp. 2491-2500. doi:10.2514/1.J050002.

[68] Michálek, J., Monaldi, M., and Arts, T., “Aerodynamic Performance of a Very High Lift Low Pressure Turbine Airfoil (T106C) at Low Reynolds and High Mach Number With Effect of Free Stream Turbulence Intensity," Journal of Turbomachinery, Vol. 134, No. 6, 2012. doi:10.1115/1.4006291.

[69] Content, C., "Méthode innovante pour le calcul de la transition laminaire-turbulent dans les codes Navier-Stokes," Ph.D. thesis, Institut Supérieur de l'Aéronautique et de l'Espace - Université de Toulouse, Toulouse, France, 2011.

[70] Menter, F. R., Langtry, R. B., Likki, S. R., Suzen, Y. B., Huang, P. G., and Völker, S., "A Correlation-Based Transition Model Using Local Variables - Part I: Model Formulation,” Journal of Turbomachinery, Vol. 128, 2006 , pp. 413-422. doi:10.1115/1.2184352.

[71] Wilcox, D. C., "Reassessment of the scale-determining equation for advanced turbulence models," AIAA Journal, Vol. 26, No. 11, 1988, pp. 1299-1310. doi:10.2514/3.10041.

[72] Kok, J., "Resolving the Dependence on Freestream Values for the k- Turbulence Model," AIAA Journal, Vol. 38, No. 7, 2000, pp. 1292-1295. doi:10.2514/2.1101.

[73] Langtry, R., and Menter, F., "Transition Modeling for General CFD Applications in Aeronautics," 43rd AIAA Aerospace Sciences Meeting and Exhibit, AIAA, American Insitute of Aeronautics and Astronautics, Reno, Nevada, USA, 2005. doi:10.2514/6.2005-522.

[74] Dopazo, C., "On conditioned averages for intermittent turbulent flows," Journal of Fluid Mechanics, Vol. 81, No. 3, 1977, pp. 433-478. doi:10.1017/S0022112077002158.

[75] Cambier, L., Heib, S., and Plot, S., "The Onera elsA CFD software: input from research and feedback from industry," Mechanics \& Industry, Vol. 14, No. 3, 2013, pp. 159-174. doi:10.1051/meca/2013056.

[76] Benoit, C., Péron, S., and Landier, S., "Cassiopee: A CFD pre- and post-processing tool," Aerospace Science and Technology, Vol. 45, No. Supplement C, 2015, pp. 272 - 283. doi:10.1016/j.ast.2015.05.023.

[77] Hunter, J. D., "Matplotlib: A 2D graphics environment," Computing In Science \& Engineering, Vol. 9, No. 3, 2007, pp. 90-95. doi:10.1109/MCSE.2007.55. 